\title{
DEM-Based Approach for the Modeling of Gelation and Its Application to Alginate
}

\author{
Philipp Nicolas Depta,* Pavel Gurikov, Baldur Schroeter, Attila Forgács, József Kalmár, Geo Paul, \\ Leonardo Marchese, Stefan Heinrich, and Maksym Dosta
}

Cite This: J. Chem. Inf. Model. 2022, 62, 49-70

\section{Read Online}

ACCESS | 네 Metrics \& More | 回国 Article Recommendations | SI Supporting Information

ABSTRACT: The gelation of biopolymers is of great interest in the material science community and has gained increasing relevance in the past few decades, especially in the context of aerogelslightweight open nanoporous materials. Understanding the underlying gel structure and influence of process parameters is of great importance to predict material properties such as mechanical strength. In order to improve understanding of the gelation mechanism in aqueous solution, this work presents a novel approach based on the discrete element method for the mesoscale

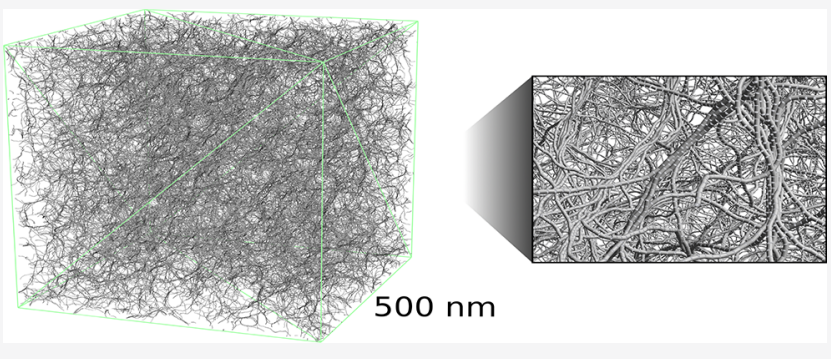
for modeling gelation of hydrogels, similarly to an extremely coarse-

grained molecular dynamics (MD) approach. For this, polymer chains are abstracted as dimer units connected by flexible bonds and interactions between units and with the environment, that is, diffusion in implicit water, are described. The model is based on Langevin dynamics and includes an implicit probabilistic ion model to capture the effects of ion availability during ion-mediated gelation. The model components are fully derived and parameterized using literature data and theoretical considerations based on a simplified representation of atomistic processes. The presented model enables investigations of the higher-scale network formation during gelation on the micrometer and millisecond scale, which are beyond classical modeling approaches such as MD. As a model system, calcium-mediated alginate gelation is investigated including the influence of ion concentration, polymer composition, polymer concentration, and molecular weight. The model is verified against numerous literature data as well as own experimental results for the corresponding Ca-alginate hydrogels using nitrogen porosimetry, NMR cryoporometry, and small-angle neutron scattering. The model reproduces both bundle size and pore size distribution in a reasonable agreement with the experiments. Overall, the modeling approach paves the way to physically motivated design of alginate gels.

\section{INTRODUCTION}

1.1. Motivation. In the past few decades, the preparation and applications of aerogels-lightweight open nanoporous materials-have gained significant scientific curiosity in the material science community. A large and growing body of the literature has emerged on the preparation of biopolymer-based hydrogels and their conversion into the corresponding aerogels. ${ }^{1-3}$ While the influence of the subsequent steps in the aerogel processing (solvent exchange and supercritical drying) on the final aerogel properties are reasonably well-understood, ${ }^{4-7}$ it still remains a challenging task to relate gelation conditions and aerogel textural properties. Although the molecular picture of the gelation is elucidated for many biopolymers, the emergence of mesostructures (fibrils, bundles, primary particles, and porous space) is to a large extent unclear. This lack of knowledge is evident since the overwhelming majority of publications almost solely rely on empirical protocols. $^{8}$ Since rapid further development through trial and error tests is associated with a lot of effort and costs, a rational physically motivated design of biopolymer aerogels would greatly advance the field.
1.2. State of the Art. Several numerical methods have been developed to study the gelation process at the microscale with the aim to analyze structural, mechanical, thermal, or electrical properties. Various simulation approaches exist for the modeling of structural formation. In addition to the widely used molecular dynamics (MD) approach, various coarse-grained sphere approaches exist, such as dissipative particle dynamics (DPD), ballistic cluster-cluster aggregation, diffusion-limited cluster(-cluster) aggregation (DLCA), and reaction-limited cluster aggregation. One of the central questions addressed with such simulations was to analyze the influence of various parameters such as density, ${ }^{9}$ polymer persistence length, ${ }^{10}$ cooling rate, ${ }^{11}$ particle roughness, or short-ranged noncentral forces ${ }^{12}$ on structural formation.

Received: September 3, 2021

Published: December 22, 2021 
The increase in computational performance of modern computer systems has allowed to increase dimensions of the investigated systems. While in the early 90 s, the box size was in the range of $200 \AA{ }^{9}$ current application of MD to investigate mechanical properties allows to reach the range of 30-100 nm. ${ }^{13-15}$ Alternative methods such as DLCA allow to reach larger dimensions in the range of $200 \mathrm{~nm},{ }^{16}$ however, containing a large number of simplifications. More accurate techniques, such as DPD, which was initially proposed by Hoogerbrugge and Koelman, ${ }^{17}$ have been applied to structural formation phenomena $^{18}$ and can typically be applied on a smaller domain in the range of $400 \AA .{ }^{19}$

Simulation studies described above have taken place in the context of MD and been limited to scales in length and time permitting only the simulation of a few polymer fibers. To tackle such scale limitations and to investigate structural formation during the gelation of polymers, mesoscale models can be formulated. One of the most promising approaches, which can be used as a further abstraction level, is the discrete element method (DEM). DEM, MD, DPD, and several others provide similar frameworks for solving Newton's equation of motion for discrete objects interacting with each other and the surrounding. The main difference between them is the level of abstraction and application scale. In either case, explicit time stepping is used for time integration and contact detection is performed, for which interaction forces are then calculated. Additional forces through bonded interaction of objects, "external" forces such as gravity, forces resulting from the thermodynamic ensemble, and field approaches/long-range forces are often included. The interested reader is referred to Berendsen. ${ }^{20}$ There exist attempts to apply DEM for similar problems related to the topic of gel microstructures; however, most of them were focused on mechanical behavior. Guesnet et al. ${ }^{21}$ used DEM to analyze mechanical behavior of precipitated silica. In their study, the simulation domain was on the $\mu \mathrm{m}$ scale. There also exist applications of DEM on the macroscopic scale; however, here, the material microstructure is not properly described. ${ }^{22}$ Additionally, micromechanical modeling of similar structures has been performed in the context of finite element modeling and beam theory. ${ }^{23,24}$

In the context of the alginate model system, a variety of simulation approaches have been applied to better understand the system, which were typically centered around $\mathrm{MD}$ and density functional theory (DFT). The original "egg-box" model for ion-mediated gelation was proposed by Grant et al. ${ }^{25}$ and has been the basis over the past decades. ${ }^{26}$ Modifications of the original egg-box model have been proposed in the following years concerning, for example, its molecular structure. ${ }^{29}$ Furthermore, works have been performed concerning ion acceptance during gelation using $\mathrm{MD}$ and $\mathrm{DFT},{ }^{28,29}$ (cross)linkage of multiple poly-G chains using $\mathrm{MD},{ }^{30,31}$ alternating compositions of polymer fibers using $\mathrm{MD},{ }^{32,33}$ and more. On larger scales, for example, Brownian dynamics and Monte Carlo models have been attempted to gain insights on polymer stiffnesses and persistence lengths. ${ }^{34}$ This work builds upon many of these contributions for parameterization of the proposed mesoscale gelation model.

\section{MODEL SYSTEM: ALGINIC ACID}

Alginate is an umbrella term for a large family of polysaccharides extracted primarily from farmed brown algae. Alginate is a linear binary copolymer of 1-4-linked residues of mannuronic (M) and guluronic $(\mathrm{G})$ acids. The residues are arranged in a blockwise pattern with homopolymeric regions of $\mathrm{G}$ residues (called G-blocks) and a homopolymeric region of $\mathrm{M}$ sequences (called M-blocks) interspersed by regions in which the two residues alternate. ${ }^{35}$ Commercial alginates have a weightaverage molecular weight $\left(\bar{M}_{\mathrm{W}}\right)$ of approximately $200 \mathrm{kDa}$ with a molar-mass dispersity between 1.5 and $3 .^{35}$

The mannuronic and guluronic acids are structurally very similar, the only difference is the configuration at the C-5 atom. Because of this difference, the $M$ and $G$ units adopt two different conformations of pyranose rings. This gives rise to flat ribbonlike and flexible chain conformations when mannuronic acid residues are linked to each other. In contrast, a pair of guluronic acid residues form a buckled and more rigid structure. Another common feature of mannuronic and guluronic residues is that they each bear a carboxylic group.

At a basic level, the bulk composition is characterized by the fraction of $M$ and $G$ units in a single alginate chain, $F_{M}$ and $F_{G}$, respectively. Because $F_{M}+F_{G}=1$, it is sufficient to provide a single value of the so-called mannuronate/guluronate ratio $F_{M}$ / $F_{\mathrm{G}}$ for characterizing the bulk composition. Being the result of complex biosynthetic pathways along with seasonal variations, the guluronate fraction can vary over a wide range $\left(0.20<F_{\mathrm{G}}<\right.$ 0.75 ) and may even depend on the part of the plant considered. ${ }^{35}$

At a more detailed level, the sequential structure of alginate can be characterized by fractions of diads, that is, fractions of four possible pairs MM, MG, GM, and GG. Fractions of triads, tetrads, and higher-order multads would be needed for a complete statistical description of a given alginate chain. ${ }^{8}$

Alginates with a high guluronate fraction $F_{\mathrm{G}}>0.7$ and an average G-block length of a few tens of residuals yield stable gels with superior mechanical strength and a lower degree of syneresis compared to M-rich alginates. Because gelling abilities of alginate positively correlate with the mannuronate/ guluronate ratio $F_{M} / F_{G}$, or the $M-G$ ratio for short, industrial alginates are often broadly classified as low-G and high-G alginates. $^{8}$

Compared with other gelling polysaccharides, the binding of di- and trivalent cations eventually resulting in the gel formation is the most striking feature of alginate as a polyanion. The most studied system is a calcium-cross-linked alginate hydrogel. In the simplest protocol, the hydrogel is obtained when aqueous sodium alginate is poured into calcium salt solution.

The detailed quantitative mechanism of metal binding and gelation still remains a subject of scientific debate. At a qualitative level, binding between $\mathrm{M}^{2+}$ and two adjacent $\mathrm{G}$ units in a single alginate chain occurs at low cation concentrations. A geometrical nest-like configuration of the $\mathrm{G}-\mathrm{G}$ block favors interactions of $\mathrm{M}^{2+}$ with carboxylate and hydroxyl groups of guluronate. The $M-M$ and $M-G$ blocks adopt much flatter conformations so that they are involved in the complexation only at higher cation concentrations. As the concentration of $\mathrm{M}^{2+}$ further increases, dimerization of $\mathrm{G}-$ $\mathrm{M}^{2+}-\mathrm{G}$ sites takes place yielding zigzag-shaped junction zones. As a result, the chains are zipped into clusters by the autocooperative binding and form what is called "egg-box" junctions. Subsequent intrachain association results in lateral and coil-like multimers which serve as building blocks for fibril-like structures of the emerging gel phase. ${ }^{36}$

Depending on the cation concentration, the $\mathrm{M}-\mathrm{G}$ local composition, and the length of alginate chains, the intrachain association can be governed by either hydrogen bonding or by electrostatic interactions and leads to multimers with different 
types of agglomeration: lateral association, chain coiling, and entanglement. $^{32}$ Small-angle X-ray scattering (SAXS) and nuclear magnetic resonance (NMR) structural studies suggest that multimers composed of a few tens of polymer chains form already with substoichiometric amounts of $\mathrm{M}^{2+}$, that is, when only a small fraction of G-blocks is occupied. In the materials science and in particular in the aerogel science, gelation is often performed in an excess of cations by dripping alginate solution into a large batch with metal salt solution (known as diffusion setting method). Available SAXS studies of Ca-alginate gels under such conditions indicate that alginate gels are made up of nanosized fibrils of a few nanometers in diameter.

The association character in the presence of calcium ions is found to depend on the chain composition. While $M$ residues operate as elasticity moderators promoting the lateral association of dimers and high-order clusters in M-rich chains, the association of G-rich zones occurs primarily through the zipping mechanism. Therefore, heteropolymeric chain compositions can form stiff zones connected by flexible junctions. ${ }^{32}$

As both $\mathrm{G}$ and $\mathrm{M}$ units are involved in the formation, we define the degree of cross-linking $f$ as follows

$$
f=\frac{\left[\mathrm{Ca}^{2+}\right]}{[\mathrm{G}]+[\mathrm{M}]}
$$

where $\left[\mathrm{Ca}^{2+}\right]$ is the molar concentration of calcium cations in the gel and $[\mathrm{G}]$ and $[\mathrm{M}]$ are the molar concentrations of $\mathrm{G}$ and $\mathrm{M}$ units, respectively. Each unit has a molecular mass of $175 \mathrm{~g} /$ mol.

\section{MODELING APPROACH}

In this section, the modeling approach will be presented. This includes an overview of the basics, structural model, functional model, simulation procedure, and implementation details. For an overview of the model parameters, the reader is referred to Section 5.1.

3.1. Fundamentals. In this contribution, a DEM-based Langevin dynamics simulation approach is proposed for modeling of the gelation process on the micrometer scale. For this purpose, a set of polymer chains are randomly generated in the simulation domain with periodic boundary conditions. Afterward, the interaction of polymer chains is modeled to describe structural formation during the gelation process. Chains are represented as a set of spherical primary particles/ beads connected with elastic solid bonds. Each particle represents a dimer unit of the polymer chain. This strategy, often named a bonded-particle model, is widely used to compute mechanical properties of various materials. ${ }^{22,37,38}$ For the modeling of the gelation process, three main interaction mechanisms are considered in the model: diffusion of units in a solvent, interaction between units of different chains, and the interaction of units within a chain through solid bonds. Moreover, ions are implicitly considered in the model using a probabilistic approach.

3.2. Structural Model. In accordance with the properties of the model system, two polymer monomer units $G$ and $M$ were abstracted as primary building blocks, thus leading to three possible types of dimer units: GG, MM, and GM. This abstraction level is reasonable for the alginic acid system, as calcium-mediated gelation is strongly dependent on the distribution of these types and structural features, such as the egg-box model, as discussed in Section 2. However, for other polymer systems, monomers or multimers might be more appropriate. Note that for simplification, the MG combination is assumed to be equivalent to GM; therefore, only GM notation is used in the article. No orientation is modeled for the particles/ beads.

In order to generate the abstracted model of the polymer chains, a two-step process, which is schematically shown in Figure 1, has been developed. In a first step, a second-order

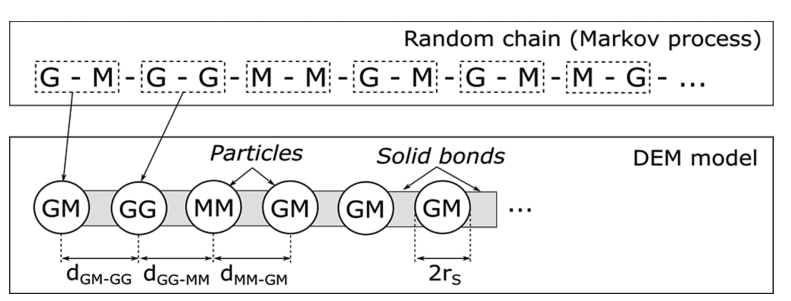

Figure 1. Generation of the structural model for polymer chains.

Markov process was used to generate the composition of polymer chains randomly. Here, $\mathrm{G}$ and $\mathrm{M}$ monomer units were generated by the Markov process. As input parameters, the probability of occurrence for all ternary combination of elements such as MMM, MMG, MGM, and so forth is specified. In a second step, the generated units are pairwise combined into the GG, MM, and GM dimer units. Each of these units is represented in the DEM model as a separate particle. Afterward, neighboring particles are connected using solid bonds with an equilibrium length $d_{i-j}$ of each bond corresponding to the equilibrium distance between unit types. This two-step process enables a direct usage of composition parameters typically available but comes at the expense of an error introduced during the combination into dimer units. This leads to an underestimation of GG and MM units over GM units, which is deemed acceptable for this model, as poly-G units are crucial for the stable gelation in any case.

The bonds connecting the individual units of the polymer chain will be further discussed in Section 3.3 and polymer chains of arbitrary length (i.e., molecular weight) can be created. Overall, the structural model has two parameter groups, the center of mass (COM) distances between the particles/beads in the chain $d_{i-j}$ and the properties of the Markov process $\left(\phi_{i j k}\right.$ indicating fraction of sequences with a composition $i, j, k$ in chain). All these model parameters are given in Section 5.1.

3.3. Functional Model. The motion of each DEM particle representing a dimer unit depends on the forces and torques acting on it, which can be caused due to diffusion, existence of solid bonds making up the polymer chains, and interaction with other particles. The translational motion of each particle is described by Newton's equation of motion as

$$
\frac{\mathrm{d} v}{\mathrm{~d} t}=\frac{1}{m}\left(F_{\mathrm{dif}}+\sum_{i}^{N_{\mathrm{b}}} F_{\mathrm{bond}, i}+\sum_{i}^{N_{\mathrm{c}}} F_{\mathrm{pp}, i}\right)
$$

where $m$ is the mass of the particle (i.e., dimer unit neglecting the increase through binding of a calcium ion), $\mathrm{d} v / \mathrm{d} t$ is its acceleration, $F_{\text {dif }}$ is the force describing diffusion, $F_{\text {bond }}$ is the force caused by solid bonds making up the polymer chains, $F_{\mathrm{pp}}$ is the interaction force acting between two unbonded particles, $N_{\mathrm{b}}$ is the number of solid bonds connected to this particle, and $N_{\mathrm{c}}$ is the number of unbonded contacts. According to the linearly sequential structural model of polymer chains, the maximal number of solid bonds per particle is two. Time integration of the equation of motion can then be performed using a leap-frog 
algorithm. Implementation details will be provided in Section 3.7.

3.3.1. Diffusion Model. In order to properly model the thermodynamics of the system and enforce the desired canonical ensemble, our previously developed diffusion model was used. ${ }^{39}$ This model is based on Langevin dynamics and allows us to describe anisotropic translational and rotational diffusion. Furthermore, this model provides a procedure to determine diffusion coefficients using MD based on a molecular reference structure. This is especially useful for highly anisotropic molecules. For this study, the diffusion model was simplified and only isotropic translational diffusion was considered. The simplification not only permits larger time steps but also simplifies calculations significantly, as no coordinate transformations are required. The forces due to diffusion for each degree of freedom $i \in\{x, y, z\}$ are $^{39}$

$$
F_{\mathrm{dif}, i}=-c_{\mathrm{dis}, i} \nu_{i}+F_{\text {fluct }, i} \xi_{i}
$$

where $v$ is the velocity and $\xi$ are normally distributed random numbers (zero mean and unit variance). The dissipative drag coefficients $c_{\mathrm{dis}}$ and fluctuating coefficients of force $F_{\text {fluct }}$ are defined as

$$
\begin{aligned}
& c_{\text {dis }, i}=\frac{m}{\Delta t}\left(1-\mathrm{e}^{-\frac{6 \pi \eta r_{\mathrm{s}, i}}{m} \Delta t}\right) \\
& F_{\text {fluct }, i}=\frac{1}{\Delta t} \sqrt{m k_{\mathrm{B}} T\left(1-\mathrm{e}^{-2 \frac{6 \pi \eta r_{\mathrm{s}, i}}{m} \Delta t}\right)}
\end{aligned}
$$

where $m$ is the mass, $\Delta t$ is the simulation time step, $\eta$ is the dynamic viscosity of the fluid, $k_{\mathrm{B}}$ is the Boltzmann constant, $T$ is the temperature, and $r_{s}$ is the Stokes radius. The derivation of the diffusion model and equations are provided in ref 39. All model parameters are given in Section 5.1.

3.3.2. Polymer Bond Model. A linear elastic bond model was implemented to describe the polymer fibers and interaction between neighboring objects within the fiber. Two degrees of freedom are acted upon by the model, namely, the distance between neighboring objects and the curvature of the fiber, described by the angle between two consecutive bonds. All the remaining degrees of freedom, such as torsional deformation of the fiber, are free, as no orientation of the particles is modeled. No bond breakage is modeled, which is reasonable as the energy stored in the covalent $\mathrm{C}-\mathrm{O}-\mathrm{C}$ bonds connecting the dimer units is significantly larger than all other kinetic and potential energies in the system.

The deformation of a bond leads to a force acting in the normal direction $F_{\text {bond,N }}$, calculated as

$$
F_{\text {bond, } \mathrm{N}}=k_{\text {bond, } \mathrm{N}} \cdot \Delta d
$$

where $k_{\mathrm{bond}, \mathrm{N}}$ is the bond normal stiffness and $\Delta d$ is the bond deformation relative to the equilibrium distance $d_{\text {eq }}$ between COM of the objects (see the Structural Model). This force acts on both particles connected by the bond. Additionally, a bending torque is generated due to transverse deformation of the polymer chain. The bending torque $T_{\text {bond }}$ depends on the relative angle between each two adjacent bonds $\alpha_{\text {rel }}$, deviating from a straight fiber and the bending stiffness $k_{\text {bond,T. It is }}$ calculated as

$$
T_{\text {bond }}=k_{\text {bond, } \mathrm{T}} \cdot \alpha_{\text {rel }}
$$

The bending torque is converted to forces acting on the three particles connected by the two bonds according to the current length of the bonds as a lever arm. As a result of this formulation, the larger the distances between two particles are $\left(d_{\text {eq }}\right)$, the lower are the resulting forces as a function of angular displacement. This inherently leads to the desired stiffnesses of the model system described in Section 2. In the context of this model, universal $k_{\text {bond,N }}$ and $k_{\text {bond,T }}$ were derived for all component combinations of the model.

3.3.3. Interaction Model. As an effective interaction potential for the unbonded interaction of particles, a Lennard-Jones (LJ) potential was chosen. This choice is to some extent arbitrary, and other potential shapes are certainly conceivable. This choice merely presents one of the simplest potential functions comprising an attractive behavior at long distances, repulsive behavior at short distances, and defined potential minimum to effectively model the calcium-mediated binding of, for example, two GG dimers in the model system. Note that for an explicit ion and charge distribution model, a Coulomb potential would be more appropriate. The basic LJ potential and the resulting force are calculated as

$$
\begin{aligned}
& U_{\mathrm{LJ}, \text { base }}=\epsilon\left(\left(\frac{d_{\mathrm{m}}}{d}\right)^{12}-2\left(\frac{d_{\mathrm{m}}}{d}\right)^{6}\right)+U_{\text {cor }} \\
& F_{\mathrm{pp}}=-\nabla U_{\text {LJ,base }}=12 \frac{\epsilon}{d_{\mathrm{m}}}\left(\left(\frac{d_{\mathrm{m}}}{d}\right)^{13}-\left(\frac{d_{\mathrm{m}}}{d}\right)^{7}\right)
\end{aligned}
$$

where $\epsilon$ is the scaling factor defining the potential well depth, $d_{\mathrm{m}}$ is the equilibrium distance and location of the potential minimum, and $U_{\text {cor }}$ is a potential correction to ensure zero potential at cutoff to avoid discontinuities in the context of the global interaction potential and postprocessing. Overall, three corrections were applied:

- to reduce computational complexity, a cutoff $d_{\text {cut }}$ between $2.1-2.5 \times d_{\mathrm{m}}$ was used in agreement with the literature ${ }^{40}$ and the potential shifted to zero at the cutoff using $U_{\text {cor }}$. Note that this leaves the force unchanged with a maximum of $1.2 \times 10^{-12} \mathrm{~N}$ at cutoff. Additionally, the force could be switched to zero employing approaches such as in ref 41 ;

- to avoid numerical problems due to the singularity at zero distance, especially during equilibration runs, a maximum gradient $-\nabla U_{\max }=F_{\max }$ was specified, which results in a linearly increasing potential after $-\nabla U_{\max }$ is exceeded;

- to consider availability of calcium, the presence of ions at contact has been considered using a probabilistic model (see the next paragraph). Importantly, note that in the case when no ion is available at a contact, the potential was set to zero above $d_{\mathrm{m}}$ and shifted to zero at $d_{\mathrm{m}}$. As a consequence, the resulting force is the negative gradient of the potential and represents only repulsive interaction.

3.3.4. Ion Model. During the gelation of alginate, the availability of calcium is of critical importance. Three strategies to consider ions were designed capturing different levels of abstraction and consequently different physics of gelation processes. The basic model (IM0) assumes ions to be fully saturated over the entire system and readily available for any interaction occurring. This model represents the full homogeneous case and produces the most uniform and densely connected gels.

The next model (IM1) describes the availability of ions and the following attractive binding as a random process due to its spatial probability in a simplified fashion (see Figure 2). 


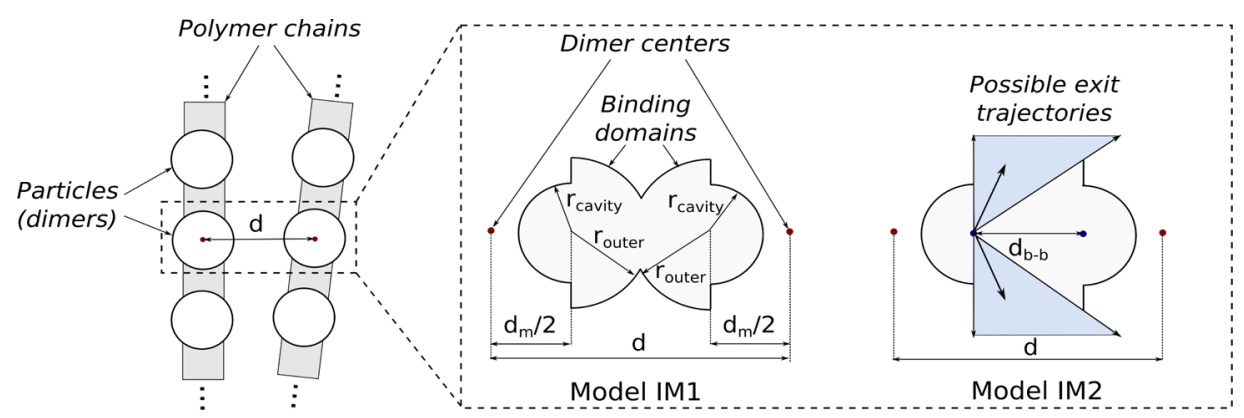

Figure 2. Ion models IM1 and IM2.

Depending on calcium concentration, a limited global number of ions are available in the simulation domain and the likelihood of one of these ions being at the binding site in each time step is modeled as

$$
p_{\text {bind }}=\frac{V_{\text {bind }}}{V_{\text {tot }}} \cdot N_{\text {avail,ions }}
$$

where $V_{\text {tot }}$ is the total volume of the simulation domain, $N_{\text {avail,ions }}$ is the number of available (i.e., unbound) ions, and $V_{\text {bind }}$ is the volume of the binding site, that is, the volume occupied by the ion for the attractive interaction to take place, which is a function of distance, interaction pair, and time step. Such a model is especially motivated by the spatially defined and confined nature of the ion-mediated zipping mechanism in the context of the "egg-box" interaction of alginate. $V_{\text {bind }}$ and the affinity of a calcium ion depend among others on the conformation of the interacting molecules, distance between them, (resulting) charge distribution (especially due to the negatively charged alginic acid dimers), and thermodynamic effects (i.e., diffusion). As a result, the potential well (minimum) occupied by the ion has a different shape, size, and potential depth. In order to understand this in its entirety, DFT simulation would be necessary, which goes beyond the scope of this work. In order to estimate and capture this effectively in a simplified fashion, the following model was formulated. Each molecule of a pairwise interaction has its own binding volume, which is independent from each other when spatially separated and overlapping when in proximity (see Figure 2). The center of the binding volume for each molecule is located at half the equilibrium distance (location of potential well) of the interaction and is simplified as spherical with an electrostatic $r_{\text {ion }}$ and a diffusive/thermodynamic component $r_{\text {dif }}$, which is time step-dependent. Depending on the conformation of the molecules, the binding volume can extend into cavities of the molecule (e.g., to capture the "eggbox" cavity) by $r_{\text {cavity. }}$. While the outer half-sphere of the binding volume $r_{\text {outer }}$ is the sum of $r_{\text {ion }}$ and $r_{\text {diff }}, r_{\text {cavity }}$ does not have a diffusive component and is determined solely by the conformation. As the binding volume can be closed off by the interacting molecules, a minimum gap $d_{\text {gap,min }}$ can be specified in the model, below which the binding volume is inaccessible from the outside. The binding model IM1 assumes that once an ion binds to a molecule (within cutoff to another molecule), it will remain bound to this interaction as long as it exists, that is, the distance between interacting molecules remains below interaction cutoff. Consequently, the overall binding probability over time is dependent on the cutoff distance chosen for the interaction model. In order to address this, the third model was formulated. All model parameters are given in Section 5.1.
The last model (IM2) relaxes these issues by modeling the unbinding probability of the calcium ions (see Figure 2), consequently leading to a dynamic equilibrium of binding and unbinding of ions. The unbinding probability of an ion from a molecule is a function of the binding energy, temperature, and geometric constraints-each of which are described and modeled next. The exact binding energy $U_{\text {ion,bind }}$ of an ion at (one) molecule is as discussed earlier complicated to be determined. In order to estimate $U_{\text {ion,bind, }}$ it is assumed being equal to half the interaction potential $\epsilon$ as it is bound primarily at one molecule and effects of the interaction partner will be captured in the geometric constraints. In the employed canonical ensemble, the probability of the kinetic energy of the ion being larger than a critical energy (e.g., binding energy with the molecule) can then be determined from the MaxwellBoltzmann distribution at the respective system temperature $T$. This probability is further lowered by geometric constraints, being that the ion can only leave away from the molecule and the directional cone toward the interaction partner with its binding region has to be subtracted, leading to a surface exit ratio $c_{\mathrm{s}}$ of

$$
c_{\mathrm{s}}=\frac{1}{2 \cdot \sqrt{\left(\frac{r_{\text {outer }}}{d_{\mathrm{b}-\mathrm{b}}}\right)^{2}+1}}
$$

where $r_{\text {outer }}$ is the radius of the interaction partners binding domain and $d_{\mathrm{b}-\mathrm{b}}$ is the distance between the centers of the ionbinding sites, that is, $d_{\mathrm{b}-\mathrm{b}}=r-r_{\mathrm{m}}$. Note that $c_{\mathrm{s}}$ can never be more than 0.5 . Overall, the probability of unbinding $p_{\text {unbind }}$ is

$$
\begin{aligned}
& p_{\text {unbind }}=c_{\mathrm{s}} \cdot\left(1-\operatorname{erf}\left(\frac{v}{\sqrt{2} a}\right)-\sqrt{\frac{2}{\pi}} \cdot \frac{v}{a} \cdot \mathrm{e}^{-v^{2} / 2 a^{2}}\right) \\
& v=\sqrt{\frac{2 U_{\text {ion,bind }}}{m_{\text {ion }}}} \\
& a=\sqrt{\frac{k_{\mathrm{B}} T}{m_{\text {ion }}}}
\end{aligned}
$$

where erf is the error function, $v$ is the velocity corresponding to $U_{\text {ion,bind }}, k_{\mathrm{B}}$ is the Boltzmann constant, and $m_{\text {ion }}$ is the ion mass. When $d_{\mathrm{b}-\mathrm{b}}$ is below the minimum gap $d_{\mathrm{gap}, \min }$, it is assumed that the ion cannot unbind anymore and $p_{\text {unbind }}$ is set to zero. The unbinding probability is uncorrelated in time for the time step $10^{-13} \mathrm{~s}$ used (i.e., no inertia effects), as the critical time step for explicitly modeling the diffusive behavior of calcium is approximately $2 \times 10^{-14}$ s and consequently residual velocity contributions are below $1 \%$ (see ref 39 ).

Strictly speaking, $U_{\text {ion,bind }}$ increases as the binding regions are overlapping, leading to a decrease in $p_{\text {unbind }}$. One could model 
this using the maximum of the $U_{\mathrm{LJ}}(d)$ and $0.5 \cdot \epsilon$ and would likely get a more accurate result for $U_{\text {ion,bind. However, at the same }}$ time, $c_{s}$ increases for increasing overlaps, as the ion becomes less constrained by one molecule and its location (distribution) shifts toward the center of the pairwise interaction, thereby exposing a larger exit range. These effects are consequently opposite and exactly modeling them is nontrivial. At the same time, in the proposed model, the inaccuracy of $U_{\text {ion,bind }}$ for increasing binding region overlap is largely mitigated by $d_{\text {gap,min }}$, which causes the error being below $10 \%$ and effected region below $0.4 \AA$. In the context of this and the opposing effect of $U_{\text {ion,bind }}$ and $c_{\mathrm{s}}$ for increasing binding overlap, the simplistic model was therefore chosen.

3.4. Simulated Annealing. One of the main problems in $\mathrm{MD}$ and also this study is that relatively long simulations are required to sample the system states. This is especially wellknown and studied for the problem of protein folding. Similarly, in this model, during the gelation, many local minima of the system exist and a very long simulation time would be required to reach a state near the global minima, that is, equilibrium. In order to numerically address this problem and speed up the process of reaching equilibrium, a simulated annealing procedure is often employed. ${ }^{42}$ During this procedure, the temperature of the system is artificially increased to increase the thermokinetic energy and consequently the likelihood of leaving a local minimum, crossing a potential barrier, and coming closer to a global minimum. The annealing temperature is then stepwise or gradually decreased to the desired system temperature and different temperature profiles have been proposed in the literature.

Various simulated annealing protocols have been tested in the scope of this work. Ultimately, a periodic annealing protocol was chosen, which consists of the following: the temperature is periodically increased to the maximum temperature $T_{\mathrm{an} \text {,max }}$ and during the time interval $\tau_{\text {an,cool }}$ linearly decreased to the equilibrium temperature. This cycle repeated with the period $\tau_{\text {an,period }}$ until the time $t_{\text {anneal,finished }}$ is reached. The viscosity of the (implicit) fluid is NOT varied during this procedure, as the primary goal during simulated annealing is to overcome local potential barriers through the increase in temperature. Furthermore, changes in viscosity would impact the time step requirements negatively.

3.5. Simulation Procedure. The simulation procedure is closely related to the typically employed strategies in $\mathrm{MD}$ and consists of four steps in the following sequence:

1. System generation: For a defined box size, polymer concentration, molecular weight, and fiber composition, random instances of polymer fibers are generated and randomly placed and oriented in the box. These instances can also cross the periodic boundary conditions and may overlap. At this state, the polymer fibers are perfectly linear.

2. Relaxation: The polymer fibers are given time to relax without interacting with each other (similarly to a diluted system). During this step, the structure relaxes and goes from the artificially straight fiber state to its natural state. This step takes approximately $50 \mu$ s without annealing and can be reduced to $15 \mu$ s using annealing procedure AN1 (see Section 5.1), which was therefore employed.

3. Equilibration: The system is given time to equilibrate in order to correct overlap between the polymer fibers and let the system equilibrate to the natural state without calcium in the system. During this step, all interaction models are set to be purely repulsive and no attractive potentials are present. Equilibration is run for $5 \mu \mathrm{s}$. No annealing is employed during this step.

4. Production (Gelation): The actual production run and gelation takes place. During this step, the full interaction model with attraction is used to represent calciummediated gelation. As this step is more prone to run into local potential minima, annealing procedure 2 (see Section 5.1) and no annealing have been employed and compared.

During all stages, the equilibration progress is checked by various global properties [global interaction potential, bond potential, kinetic energy, and bond-angle correlation (BAC) (see Section 5.1)] and simulation times were chosen accordingly.

3.6. Postprocessing. The simulation results were analyzed both online during the simulation and in postprocessing. Analyzed properties of the system include the following:

- global energy $U$ in components of the potential energy due to pairwise interaction (normalized by number of dimers), bonded potential energy, and kinetic energy;

- ratio $f$ of used, available, or desired ions. Desired ions represent unbonded contacts capable of receiving an ion;

- normalized contact number $\xi_{i-j}=2 N_{\mathrm{C}, i-j} / \sqrt{N_{i} N_{j}}$, where $N_{\mathrm{C}, i-j}$ is the number of contacts between type $i$ and $j$ based on an LJ cutoff of $1.5 \mathrm{~nm}$ and $N_{i}\left(N_{j}\right)$ is the number of particles of type $i(j)$;

- coordination number both average $\bar{k}_{0.9 \mathrm{~nm}}$ and histogram fractions $\phi$ based on a reduced contact radius of $0.9 \mathrm{~nm}$ to capture only close proximity contacts. The trends of the average coordination number are inversely proportional to the specific surface of the polymer;

- spatially resolved volume fractions $\phi_{\mathrm{V}}$ of particle concentrations. The simulation domain is discretized on a regular grid of $5 \mathrm{~nm}$ into cubes and the concentration in each cell is calculated by the number of particles within the cell divided by its volume. Based on this, volume fractions of pores (empty cells) and bundles (cells with high concentration) have been calculated;

- pore size distribution (PSD) based on chord length distribution for comparison with experimental data. The chord length distribution is calculated using the Python library PoreSpy based on the previous $5 \mathrm{~nm}$ discretization with various limits as to whether a voxel is occupied or not; and

- fibril/bundle thickness (number of fibers in a bundle) and bundle diameter (maximum distance between particles in bundle cross section) for comparison with experimental data. A contact network search algorithm determines the particles in the bundle cross section for each particle in the system, while only considering the closest contact (contact distance $0.9 \mathrm{~nm}$ as for coordination number) between particles of two fibers and only passing each fiber once.

Additional information about the chord length of pores is provided in the Supporting Information.

3.7. Implementation. The proposed simulation strategy and developed models are implemented in the open-source DEM simulation framework MUSEN. ${ }^{43}$ The code was implemented in $\mathrm{C}++$ and CUDA for the simulation on graphics 
processing units (GPUs) using the CUDA Toolkit v11.2 by NVIDIA $^{44}$ with floating point precision. Due to the random component introduced by the diffusion model, no higher precision is required. For time integration, the leap-frog algorithm was used and contact detection performed using a Verlet list implementation. ${ }^{43}$ A time step of $10^{-13} \mathrm{~s}$ was used unless otherwise indicated and the simulation procedure outlined in Section 3.5 was employed. For the diffusion model, ${ }^{39}$ a separate pseudo-random-number generator was seeded with a unique seed for each particle. For the generation of random numbers, the XORWOW $W^{45}$ algorithm from the standard CUDA random number library ${ }^{44}$ was used. For random generation of orientations in system generation, the algorithm ${ }^{46}$ was used. Values were tabulated for interaction forces with a resolution of $0.01 \AA$ and for binding probabilities with a resolution of $0.1 \AA$.

\section{EXPERIMENTAL SETUP}

4.1. Preparation of Ca-Alginate Hydrogels. A jet cutter (geniaLab BioTechnologie $\mathrm{GmbH}$, type S: S1702A) was used for the production of spherical Ca-alginate hydrogel particles as described earlier. ${ }^{47,48}$ In the jet-cutting process, solution of sodium alginate from a storage tank was extruded through the nozzle. The liquid jet was cut with a rotating disc with many thin wires into small segments. The initially cylindrical segments form spherical droplets while falling directly into a constantly stirred gelation bath with calcium chloride solution (0.5, 1.0 and 5.0 wt \%). The obtained beads were immersed into fresh gelation bath for at least $12 \mathrm{~h}$ to ensure complete cross-linking. The hydrogels were either directly converted into aerogels as described elsewhere ${ }^{47,48}$ or preserved with $0.02 \mathrm{wt} \%$ sodium azide prior to NMR cryoporometry and small-angle neutron scattering (SANS) measurements.

4.2. Hydrogels: G/M Ratio with Solid-State NMR. Solidstate NMR spectra were acquired on a Bruker Avance III 500 spectrometer and a wide bore $11.7 \mathrm{~T}$ magnet with operational frequencies for ${ }^{1} \mathrm{H}$ and ${ }^{13} \mathrm{C}$ of 500.13 and $125.77 \mathrm{MHz}$, respectively. A $4 \mathrm{~mm}$ triple resonance probe, in the double resonance mode, with magic angle spinning (MAS) was employed in all the experiments. The hydrogel was directly packed into a Kel-F insert, with an internal volume of $25 \mathrm{~mL}$, and sealed with plug and screw (to suppress the escape of fluids). ${ }^{49}$ Then, the insert was fitted into a $4 \mathrm{~mm}$ rotor and submitted for the NMR experiments (see Supporting Information S1.4). The rotor was spun at an MAS rate of $5 \mathrm{kHz}$.

For the ${ }^{13} \mathrm{C}$ cross-polarization (CP) MAS experiments, the proton radio frequencies (RFs) of 55 and $28 \mathrm{kHz}$ were used for the initial excitation and decoupling, respectively. During the $\mathrm{CP}$ period, the ${ }^{1} \mathrm{H}$ RF field was ramped using 100 increments, whereas the ${ }^{13} \mathrm{C}$ RF field was maintained at a constant level. During the acquisition, the protons were decoupled from the carbons using a Spinal-64 decoupling scheme. A moderate ramped RF field of $55 \mathrm{kHz}$ was used for spin locking, while the carbon RF field was matched to obtain an optimal signal (40 $\mathrm{kHz}$ ). A CP contact time of $10 \mathrm{~ms}$ and a delay between scans of $2.8 \mathrm{~s}$ were employed. Spectra were recorded with a spectral width of $42 \mathrm{kHz}$, and $80 \mathrm{k}$ transients were accumulated at $298 \mathrm{~K}$. All chemical shifts are reported using the $\delta$ scale and are externally referenced to TMS at $0 \mathrm{ppm}$.

${ }^{13} \mathrm{C}$ CPMAS NMR spectra were fitted for quantitative deconvolution of overlapping peaks. ${ }^{50}$ The line shape of the peaks is described by Gaussian/Lorentzian line shapes. The G/
$\mathrm{M}$ ratio is determined according to the method reported previously. ${ }^{51,52}$

4.3. Hydrogels: NMR Cryoporometry. The PSD of the Ca-alginate hydrogels was measured by NMR cryoporometry. ${ }^{53-55}$ The hydrogel beads were probed in their as-prepared states. The beads were inserted into NMR tubes after carefully removing extra water from their surface. Measurements were performed in a Bruker Avance II $360 \mathrm{MHz}$ NMR instrument. Cooling was achieved with dried air supplied by BCU-05 and BSCU-05 cooling units. The Carr-Purcell-Meiboom-Gill (CPMG) spin-echo pulse sequence was applied to eliminate the broad ${ }^{1} \mathrm{H}$ signal of the solid phase during the echo time. The remaining signal intensity is proportional to the amount of liquid water confined in the porous system, which changes gradually with temperature, as water freezes or ice melts. Before every measurement, the length of the $90^{\circ}$ pulse was determined ( $c a .11$ $\mu \mathrm{s})$. The optimization of the echo time was performed by monitoring ${ }^{1} \mathrm{H}$ signal intensity in melting hydrated mesoporous silica aerogel as a reference material at 263.0 and $268.0 \mathrm{~K}^{56}$ The optimal echo time was found to be $0.90 \mathrm{~ms}$ for the Ca-alginate hydrogel samples for filtering off the fast relaxing signal of solid water. Every sample was frozen at $253 \mathrm{~K}$ before placing it into the precooled NMR instrument. Multiple melting-freezing cycles were measured between 262.2 and $275.4 \mathrm{~K}$ in $0.2 \mathrm{~K}$ steps. Temperature was calibrated using glycol and methanol. ${ }^{57}$ The sample was left for $5 \mathrm{~min}$ at a given temperature for equilibration before running the CPMG sequence. The relaxation-filtered signal intensity in the CPMG sequence is proportional to the amount of liquid water confined in the porous system. It changes gradually with temperature, as water freezes or ice melts. ${ }^{53-55}$ The melting and freezing points of liquids confined in nanometer wide spaces are lowered, as given by the modified Gibbs-Thomson equations

$$
\begin{aligned}
& \Delta T_{\mathrm{m}}=T_{\mathrm{m}}-T_{0}=-\frac{n_{\mathrm{m}} K_{\mathrm{c}}}{r_{\mathrm{p}}} \\
& \Delta T_{\mathrm{f}}=T_{\mathrm{f}}-T_{0}=-\frac{n_{\mathrm{f}} K_{\mathrm{c}}}{r_{\mathrm{p}}}
\end{aligned}
$$

In eq $15, \Delta T_{\mathrm{m}}$ is the melting point depression expressed as a difference between the phase transition temperature of the bulk $\left(T_{0}\right)$ and the confined liquid $\left(T_{\mathrm{m}}\right) . K_{\mathrm{c}}$ is the cryoporometric constant, $n_{\mathrm{m}}$ is the geometric factor describing melting, and $r_{\mathrm{p}}$ stands for the average pore radius. In eq 16, the symbols denote the corresponding parameters for freezing. The numerical values of $\Delta T_{\mathrm{m}}$ and $\Delta T_{\mathrm{f}}$ and $n_{\mathrm{m}}$ and $n_{\mathrm{f}}$ are different because melting and freezing mechanisms of liquids in confined spaces show a hysteresis in general. ${ }^{55}$ The PSD of the hydrogel sample was calculated using the methodology of Petrov and Furo. ${ }^{55}$ The measured water peak integrals (intensities) were plotted against temperature calculated as a difference $(\Delta T)$ from the freezing point of the bulk liquid phase. The freezing points of the small quantities of the bulk fluid in each sample served as experimental reference temperatures $(\Delta T=0 \mathrm{~K})$. Intensity versus $\Delta T$ data were transformed to intensity versus pore size $\left(d_{\text {pore }}\right)$ data using the modified Gibbs-Thomson equations (eqs 15 and 16). PSD curves were calculated by estimating log-normal distribution of the pore sizes. Each intensity versus $\log _{10}\left(d_{\text {pore }}\right)$ data set was fitted with a smoothing curve composed of an appropriate number of summarized error functions, and the best fit was transformed to PSD as a probability density function. This 
method has successfully been used for characterizing hydrogellike systems. ${ }^{58}$

4.4. Hydrogels: Small-Angle Neutron Scattering. Hydrogel beads were analyzed by SANS according to the previously published protocol. ${ }^{51,59}$ Briefly, the measurements were performed in a pin-hole-type instrument with a twodimensional neutron detector, and the measured scattering intensity was corrected for sample transmission, empty cell scattering, solvent scattering, detector sensitivity, and background scattering. The structural parameters of the scattering objects were determined by the mathematical analysis of the corrected $I(Q)$ curves. For the $Q$ range where both the Guinier and the Porod approximations are applicable, their combination referred to as the Beaucage model can be used for data fitting. ${ }^{60,61}$ The water content of the Ca-alginate hydrogel was exchanged to $\mathrm{D}_{2} \mathrm{O}$ for the SANS measurements. The hydrogel beads were soaked in $\mathrm{D}_{2} \mathrm{O}$ (with 0.5 wt $\% \mathrm{CaCl}_{2}$ ) for 2 weeks, while the solvent was exchanged for fresh in every 3 days.

4.5. Aerogels: Pore Size Distribution and Calcium Content. The PSD of aerogels was measured using lowtemperature $\mathrm{N}_{2}$ adsorption-desorption analysis (Nova $3000 \mathrm{e}$ surface area analyzer, Quantachrome Instruments, Boynton Beach, USA). The desorption data were analyzed by the $\mathrm{BJH}$ (Barrett-Joyner-Halenda) method. Aerogel samples (20-30 $\mathrm{mg}$ ) were degassed under vacuum at $60{ }^{\circ} \mathrm{C}$ for $6 \mathrm{~h}$ prior to analysis. Calcium content in the aerogels was routinely quantified by ICP-OES after dissolution in $\mathrm{HNO}_{3} / \mathrm{H}_{2} \mathrm{O}_{2}$ at the central laboratory (Zentrallabor Chemische Analytik, TUHH).

\section{SIMULATION STUDIES}

5.1. Estimation of Model Parameters. All model parameters were taken either from the literature and/or approximated and will be provided in this section. An overview of all model parameter can be found in Table 1 . The mass of all dimer units of the alginate system is $350 \mathrm{Da}$.

5.1.1. Structural Model. The atomistic reference structures of the dimer units were used to estimate structural model parameters. For these, the equilibrated structures from ref 32 were thankworthy, provided by the authors. From these reference structure, the distances between the COMs of all dimer units were estimated, which can be found in Table 1 . The variation in distances has a direct impact on the chain-bending stiffness leading to stiffnesses in the order of poly-G > poly-M > alternating (GM), which is consistent with the chain compositions discussed in Section 2 and the literature. ${ }^{32}$

The parameters for the second-order Markov chain determining the composition of the polymer fibers were adapted from ref 66 and corrected in order to be a valid and complete Markov chain, see the overview in Table 2 and further details in the Supporting Information. The molecular weight of polymer fibers was set to either 100 or $200 \mathrm{kDa}$ depending on the simulation case. This resulted in a polymer chain length of 285 or 571 dimers.

5.1.2. Diffusion Model. All simulation studies were carried out at an equilibrium temperature of $300 \mathrm{~K}$ (also see the Simulated Annealing) and a dynamic viscosity for water of 8.54 $\times 10^{-4} \mathrm{~Pa} \mathrm{s.}{ }^{62}$ The Stokes radius of the dimer units was estimated from the reference structure ${ }^{32}$ by the following methodology: the dimers were first oriented along their principle axes. The radius of gyration $R_{\mathrm{g}}$ was calculated for all axes and dimer types. The Stokes radius was then estimated by accounting for the shell of hydration using the relation $r_{\mathrm{s}}=r_{\mathrm{g}}$ /
Table 1. Model Parameters ${ }^{a}$

\begin{tabular}{|c|c|c|}
\hline parameter & value & literature/notes \\
\hline \multicolumn{3}{|c|}{ Natural Constants } \\
\hline Boltzmann constant $k_{\mathrm{B}}$ & $1.38 \times 10^{-23} \mathrm{~J} \mathrm{~K}^{-1}$ & \\
\hline Avogadro constant $N_{\mathrm{A}}$ & $6.02 \times 10^{23} \mathrm{~mol}^{-1}$ & \\
\hline \multicolumn{3}{|c|}{ Simulation Constants } \\
\hline time step $\Delta t$ & $10^{-13} \mathrm{~s}$ & \\
\hline \multicolumn{3}{|c|}{ Equilibrium COM Distances } \\
\hline$d_{\mathrm{GG}-\mathrm{GG}}$ & $0.92 \mathrm{~nm}$ & 32 \\
\hline$d_{\mathrm{GG}-\mathrm{MM}}, d_{\mathrm{GG}-\mathrm{GM}}$ & $0.97 \mathrm{~nm}$ & 32 \\
\hline$d_{\mathrm{MM}-\mathrm{MM}}$ & $1.01 \mathrm{~nm}$ & 32 \\
\hline$d_{\mathrm{GM}-\mathrm{GM}}, d_{\mathrm{MM}-\mathrm{GM}}$ & $1.02 \mathrm{~nm}$ & 32 \\
\hline fiber molar mass & $100-200 \mathrm{kDa}$ & \\
\hline \multicolumn{3}{|c|}{ Diffusion Model (GG/MM/GM) } \\
\hline temperature $T$ & $300 \mathrm{~K}$ & \\
\hline dynamic viscosity $\eta$ & $8.54 \times 10^{-4} \mathrm{~Pa} \mathrm{~s}$ & 62 \\
\hline dimer mass $m$ & $350 \mathrm{Da}$ & \\
\hline Stokes radius $r_{s}$ & $0.35 \mathrm{~nm}$ & 32 \\
\hline \multicolumn{3}{|c|}{ Polymer Bond Model } \\
\hline normal stiffness $k_{\text {bond, } \mathrm{N}}$ & $2.5 \mathrm{~N} \mathrm{~m}^{-1}$ & \\
\hline bending stiffness $k_{\mathrm{bond}, \mathrm{T}}$ & $2.5 \times 10^{-19} \mathrm{~N} \mathrm{~m} \mathrm{rad}^{-1}$ & 32,63 \\
\hline \multicolumn{3}{|c|}{ Interaction Model } \\
\hline shape & Lennard-Jones & \\
\hline cutoff $d_{\text {cut }}$ & $1.5 \mathrm{~nm}$ & \\
\hline gradient limit $\nabla U_{\max }$ & $\pm 500 \mathrm{~kJ} / \mathrm{mol} / \mathrm{nm}$ & \\
\hline $\mathrm{GG}-\mathrm{GG} d_{\mathrm{m}}$ & $0.6 \mathrm{~nm}$ & 33 \\
\hline GG-GG $\epsilon$ & $22 \mathrm{~kJ} / \mathrm{mol}$ & attr. + rep. ${ }^{36}$ \\
\hline $\mathrm{MM}-\mathrm{MM} d_{\mathrm{m}}$ & $0.7 \mathrm{~nm}$ & 33 \\
\hline $\mathrm{MM}-\mathrm{MM} \epsilon$ & $3 \mathrm{~kJ} / \mathrm{mol}$ & attr. + rep. ${ }^{36}$ \\
\hline $\mathrm{XX}-\mathrm{XX} d_{\mathrm{m}}$ & $0.7 \mathrm{~nm}$ & 33 \\
\hline \multicolumn{3}{|c|}{ Ion Model } \\
\hline$m_{\mathrm{Ca}^{2+}}$ & $40 \mathrm{u}=6.64 \times 10^{-26} \mathrm{~kg}$ & \\
\hline$r_{\text {ion }}$ & $1.2 \AA$ & 64 \\
\hline$r_{\text {dif }}$ & $0.3 \AA$ & 65 \\
\hline$d_{\text {gap, min }}$ & $1 \AA$ & \\
\hline GG-GG $r_{\text {cavity }}$ & $r_{\text {ion }}=1.2 \AA$ & \\
\hline $\mathrm{MM}-\mathrm{MM} r_{\text {cavity }}$ & $0 \AA$ & \\
\hline $\mathrm{XX}-\mathrm{XX} r_{\text {cavity }}$ & $0 \AA$ & \\
\hline GG-GG $r_{\text {outer }}$ & $r_{\text {ion }}+r_{\text {dif }}=1.5 \AA$ & \\
\hline $\mathrm{MM}-\mathrm{MM} r_{\text {outer }}$ & $r_{\text {ion }}+r_{\text {dif }}=1.5 \AA$ & \\
\hline $\mathrm{XX}-\mathrm{XX}$ & $0 \AA$ & \\
\hline
\end{tabular}

0.77 in each direction, which is typically used for globular proteins. Alternatively, one could study the diffusion kinetics, for example, in $\mathrm{MD}$ and get a more detailed insight concerning solvent interaction, as performed by us in ref 39 . The estimated Stokes radii were found to be between 0.29 and $0.42 \mathrm{~nm}$ (see Table 3) with an average of $0.35 \mathrm{~nm}$. Motivated by the low degree of anisotropy, as well as model simplicity and performance reasons, the Stokes radius was set to $0.35 \mathrm{~nm}$ for all dimer units.

5.1.3. Polymer Bond Model. The polymer bond model has two parameters, the normal stiffness $k_{\text {bond, } N}$ and the bending stiffness $k_{\text {bond,T }}$, which have to be determined. For the model system, both are universal for bonds between all component combinations. This leads to reasonable results, as the stiffness relation depending on the composition becomes poly-G > poly$M>$ mixed ( $G M / M G)$ and is consistent with the literature. ${ }^{32}$ The parameters could, for example, be determined using steered $\mathrm{MD}$ or an umbrella sampling approach. However, for simplicity, 
Table 2. Properties of the Second-Order Markov Chain of Alginate (Fractions of Class Specified by the Index) Adapted from Ref 66

\begin{tabular}{|c|c|c|c|c|c|c|c|c|}
\hline & \multicolumn{8}{|c|}{ main parameters } \\
\hline & $\phi_{\mathrm{GGG}}$ & $\phi_{\mathrm{GGM}}$ & $\phi_{\mathrm{GMG}}$ & $\phi_{\mathrm{GMM}}$ & $\phi_{\mathrm{MGG}}$ & $\phi_{\mathrm{MGM}}$ & $\phi_{\mathrm{MMG}}$ & $\phi_{\text {ммм }}$ \\
\hline high G $(H)$ & 0.475 & 0.050 & 0.055 & 0.050 & 0.050 & 0.055 & 0.050 & 0.215 \\
\hline \multirow[t]{3}{*}{ low G (L) } & 0.180 & 0.020 & 0.045 & 0.085 & 0.020 & 0.110 & 0.085 & 0.455 \\
\hline & \multicolumn{8}{|c|}{ resulting fractions } \\
\hline & \multicolumn{2}{|c|}{$\phi_{\mathrm{G}}$} & $\phi_{\mathrm{M}}$ & \multicolumn{2}{|r|}{$\phi_{\mathrm{GG}}$} & $\phi_{\mathrm{GM}}$ & \multicolumn{2}{|r|}{$\phi_{\mathrm{MM}}$} \\
\hline high G $(\mathrm{H})$ & \multicolumn{2}{|c|}{0.630} & 0.370 & \multicolumn{2}{|r|}{0.525} & 0.105 & \multicolumn{2}{|r|}{0.265} \\
\hline low $\mathrm{G}(\mathrm{L})$ & \multicolumn{2}{|c|}{0.330} & 0.670 & \multicolumn{2}{|r|}{0.200} & 0.130 & \multicolumn{2}{|r|}{0.540} \\
\hline
\end{tabular}

Table 3. Stokes Radii for All Dimer Units of the Alginate System in $x, y, z^{a}$

$\begin{array}{lccc} & r_{\mathrm{s}, x}(\mathrm{~nm}) & r_{\mathrm{s}, y}(\mathrm{~nm}) & r_{\mathrm{s}, z}(\mathrm{~nm}) \\ \text { GG } & 0.29 & 0.34 & 0.40 \\ \text { MM } & 0.29 & 0.41 & 0.42 \\ \text { GM } & 0.29 & 0.35 & 0.39\end{array}$

${ }^{a}$ For the model, an average of $0.35 \mathrm{~nm}$ was used.

an optimization approach using experimental data was chosen. As an optimization goal for the bending stiffness, the BAC function $^{63}$ and the resulting persistence length $l_{\mathrm{p}}$ were calculated and tuned to the results of ref 32 , representing the polymer fiber's shape. The BAC is defined as the normalized scalar product of bond vectors $\vec{b}$ at increasing distances in the chain and decays exponentially with increasing distance ${ }^{32,63}$

$$
\begin{aligned}
& \operatorname{BAC}(i)=\left\langle\frac{\vec{b}_{j} \cdot \vec{b}_{j+i}}{\left\|\vec{b}_{j}\right\| \cdot\left\|\vec{b}_{j+i}\right\|}\right\rangle=\langle\cos (\theta)\rangle \\
& \operatorname{BAC}(x)=\exp \left(\frac{-x \sigma}{l_{\mathrm{p}}}\right)
\end{aligned}
$$

The results of ref 32 for a saturated calcium alginate system predict an $l_{\mathrm{p}}$ between 20 and $30 \mathrm{~nm}$ for poly-M and poly- $\mathrm{G}$ fibers and for alternating components, an $l_{\mathrm{p}}<5-10 \mathrm{~nm}$. As the model system has a high poly- $\mathrm{G}$ content, the optimization target was set to be between $l_{\mathrm{p}}=15-20 \mathrm{~nm}$. As an optimization target parameter for the normal stiffness, the bond strain was used. As no data were available for fine-tuning, the goal was to have a reasonably stable bond (maximum strain $<0.25$ ) and near-zero strain mean, as well as no negative impact on the required time step. After energy considerations to determine a parameter range, free diffusion experiments were run with 100 polymer fibers of 571 dimer length over a duration of $50 \mu \mathrm{s}$ with a time step of $10^{-13}$ and $5 \times 10^{-14} \mathrm{~s}$ for convergence checking. During these runs, the polymer fibers did not interact with each other, representing a dilute system. As a final parameter set, the values of $k_{\text {bond, } \mathrm{N}}=2.5 \mathrm{~N} \mathrm{~m}^{-1}$ and $k_{\text {bond,T }}=2.5 \times 10^{-19} \mathrm{~N} \mathrm{~m} \mathrm{rad}^{-1}$ were found. For this parameter set, the persistence length $l_{\mathrm{p}}$ is $16 \mathrm{~nm}$ and the bond strain has an average value of 0.004 , a minimum value of -0.16 , and a maximum value of 0.22 . Note that while the bond model and its parameters permit flexible movement and bending of the fibers, the formation of knots appears unlikely in the context of persistence lengths of $16 \mathrm{~nm}$ and none could be visually observed. However, for lower persistence lengths (e.g., for alternating $\mathrm{G}$ and $\mathrm{M}$ units), as well as lower molecular weights, such effects might be more notable.
5.1.4. Interaction Model. The interaction model and its parameter are most critical in controlling the gelation process. The parameters for it were largely taken from various literature sources and beyond that chosen on an educated-guess basis. Consequently, the model and its parameters do not claim absolute correctness by any means-they merely present a starting point for modeling and a more detailed investigation, for example, through umbrella sampling or steered $\mathrm{MD}$, is advisable, but beyond the scope of what this work is meant to show.

The calcium-mediated gelation of alginic acid is, as largely accepted in the literature, dominated by dimerization of poly-G fiber regions in which dimers interact through an "egg-box" association mediated by one calcium ion, ${ }^{25}$ followed by a lateral association and zipping mechanism as described by many authors, for example, refs 32 and 33. Due to the flatter structure of poly-M and GM-areas, these regions aggregate less readily. $^{25,32,33}$ However, poly-M regions are also said to associate through dimerization at low ion concentrations. ${ }^{32}$ In this context, Fang et al. ${ }^{36}$ measured the binding enthalpy of calcium alginate with a $\mathrm{G}$ content of $46 \%$ to be $\Delta H=-11.6 \mathrm{~kJ} / \mathrm{mol}$ and for a system with a $\mathrm{G}$ content of $64 \%$ to be $\Delta H=-15.0 \mathrm{~kJ} / \mathrm{mol}$ (Gibb's free energy in both cases $\Delta G=-23.0 \mathrm{~kJ} / \mathrm{mol}$ ). Other works on the modeling side have found slightly lower Gibb's free energies associated with the "egg-box" association in the range of $\Delta G=-35$ to $-60 \mathrm{~kJ} / \mathrm{mol}$.

These effects are captured in the interaction model through the binding energy and equilibrium distance dependent on the kind of unit interaction, as well as ion availability. In the context of binding energies, the experimentally measured binding enthalpies by Fang et al. ${ }^{36}$ present the starting point for this work. When extrapolating the G-content to 0 and 1 , this leads to an approximate binding enthalpy of $-22 \mathrm{~kJ} / \mathrm{mol}$ for pure poly- $\mathrm{G}$ and $-3 \mathrm{~kJ} / \mathrm{mol}$ for pure poly-M systems, which were used as the binding enthalpy of GG-GG and MM-MM, respectively. As it is generally understood that these are the dominating interactions for association, all other component combinations were set to be repulsive only with a scaling factor $\epsilon=-1 \mathrm{~kJ} / \mathrm{mol}$.

In order to estimate the equilibrium distance between the COM of binding units (mediated by calcium ions), the work by ref 33, which investigated the sequence-dependent association of alginate using $\mathrm{MD}$, was used as guidance. The equilibrium distances $r_{\mathrm{m}}$ used are $6 \AA$ for GG-GG association and $7 \AA$ for MM-MM association and all others. Based on this, the cutoff distance for interaction was set to be $1.5 \mathrm{~nm}\left(2.1-2.5 \times d_{\mathrm{m}}\right)$ in agreement with the literature. ${ }^{40}$ Note that the maximum force at cutoff is $1.2 \times 10^{-12} \mathrm{~N}$.

Lastly, the gradient limit $\nabla U_{\max }$ was set to $\pm 500 \mathrm{~kJ} / \mathrm{mol} / \mathrm{nm}$, which will be understood in the context of the critical time step (see next section), and is reasonably large to only come into 
effect very rarely during equilibration. An overview of all parameters can be found in Table 1 .

5.1.5. Critical Time Step. In order to estimate the simulation time step necessary for convergent and numerically stable behavior of the model, the critical time step $\tau_{\text {crit }}$ was estimated for all three model components. Generally speaking, the critical time step is a complex eigenvalue problem of the structure's natural frequencies being dependent on the excitation and dampening of the diffusion model. In order to simplify the solution, the model components were analytically investigated separately and additionally, a convergence study was performed. For the diffusion model, the critical time step was estimated according to ref 39 for the simplified case of free diffusion. For the normal component of the bonded interaction and the unbonded interactions, the critical time step was estimated based on the oscillation period for the corresponding two-massspring system and maximum stiffness in the system. For the bending component of the bond model, the critical time step was estimated based on the oscillation period of a three-massangular-spring system near its linear equilibrium state (linear fiber) with equal spacing $d_{\text {bond,min }}=0.92 \mathrm{~nm}$ between the masses. As all unit components have equal mass $m=5.812 \times 10^{-25} \mathrm{~kg}$, the critical time steps can be calculated as

$$
\begin{aligned}
\tau_{\text {crit }}= & \min \left(\tau_{\text {crit }}^{\mathrm{dif}}, \tau_{\text {crit }}^{\text {bond,N }}, \tau_{\text {crit }}^{\text {bond, } \mathrm{T}}, \tau_{\text {crit }}^{\mathrm{pp}}\right) \\
= & \min \left(\frac{m}{6 \pi \eta r_{\mathrm{s}}}, 2 \pi \sqrt{\frac{m}{k_{\text {bond,N }}}}, 2 \pi \sqrt{\frac{m d_{\text {bond,min }}^{2}}{2 k_{\text {bond, } \mathrm{T}}}}, 2 \pi\right. \\
& \left.\sqrt{\frac{m}{k_{\mathrm{pp}}}}\right)
\end{aligned}
$$

over all unit-type combinations, where $\tau^{\mathrm{dif}}$ is the critical time step of the diffusion model, $\tau^{\text {bond }}$ is the critical time step of the bond model, $\tau^{\mathrm{pp}}$ is the critical time step of the unbonded particleparticle interaction model, $r_{\mathrm{s}}$ is the Stokes radius, $\eta$ is the dynamic viscosity of the fluid, $k_{\mathrm{bond}, \mathrm{N}}$ is the normal bond stiffness, $k_{\mathrm{bond}, \mathrm{T}}$ is the bending bond stiffness, and $k_{\mathrm{pp}}$ is the stiffness of the unbonded interaction between units. Based on this, the critical time step is $1.0 \times 10^{-13} \mathrm{~s}$ for the diffusion model, $3.0 \times 10^{-12} \mathrm{~s}$ for the normal stiffness of the bond model, $6.2 \times$ $10^{-12} \mathrm{~s}$ for the bending stiffness of the bond model, and $7.3 \times$ $10^{-13} \mathrm{~s}$ for the interaction model with a gradient limit of \pm 500 $\mathrm{kJ} / \mathrm{mol} / \mathrm{nm}$.

Typically, for bonded interactions, a time step of $0.2 \cdot \tau_{\text {crit }}$ should be used for numerical stability, ${ }^{67}$ which would be fulfilled for with a time step of $10^{-13} \mathrm{~s}$. This time step is also at the advisable limit of the diffusion model, leading to an error of $8.0 \%$ in the root-mean-squared displacement. ${ }^{39}$ Consequently, this time step was chosen and cross-checked in convergence runs with a time step of $5 \times 10^{-14} \mathrm{~s}$, which produced no notable differences.

5.1.6. Ion Model. The ion model and its parameters are critical in describing the availability of ions and probability of the attractive interactions necessary for gel formation and breakage. Consequently, the ion model has an impact on both the gelation dynamics and the steady-state structure. The ion-saturated model IM0 does not require any parameters and represents the most immediate and homogeneous release of ions for gelation. The ion-binding model IM1 requires multiple parameters in relation to the ions used $\left(\mathrm{Ca}^{2+}\right)$, interaction partners, and time step, which will be discussed next, starting with the ion-related parameters. In order to estimate the electrostatic component of the binding site volume, an ionic radius $r_{\text {ion }}$ of $1.2 \AA$ for calcium was used, which is in the region of $1.0-1.4 \AA$ according to Shannon. ${ }^{64}$ Note that hydration effects of the calcium ion were neglected. In light of strong dissociation energies of water molecules from the shell of hydration $(\sim 21.9 \mathrm{kcal} / \mathrm{mol}$ for the first water molecule and $\sim 26.3 \mathrm{kcal} / \mathrm{mol}$ for the second according to ref 68), future studies might investigate these effects through the increased values of the effective ionic radius. In addition to the electrostatic component, a diffusive contribution $r_{\text {dif }}$ of $0.3 \AA$ for a time step of $10^{-13} \mathrm{~s}$ was added at the outer half-sphere of the interaction region. This contribution was estimated from the average of the diffusive length based on the diffusion coefficient from ref 65 (0.16 $\AA$ ) and mean velocity characteristic length $(0.43 \AA)$ and is consequently time step-dependent. In the case of the "eggbox" GG-GG interaction, the electrostatic component of the binding volume extends into the molecule from the equilibrium distance by $r_{\text {cavity }}=r_{\text {ion }}=1.2 \AA$, which is reasonable in regard to the conformation of the GG dimer and its cavity. In the case of the flatter MM-MM interaction, such a cavity does not exist and only the outer half-sphere is considered being the sum of the ionic and diffusive radius $r_{\text {outer }}=r_{\text {ion }}+r_{\text {dif }}=1.5 \AA$. Additionally, during binding, an enclosure around the ion forms, and a minimum gap of $d_{\text {gap,min }}=1 \AA$ has to be between the molecules in addition to their equilibrium distance. The proposed value is slightly lower than the ionic radius $r_{\text {ion }}$ to account for possible conformational changes in the molecules. The ion-unbinding model IM2 does not require any additional parameters besides the ion mass being $m_{\mathrm{Ca}^{2+}}=40 \mathrm{u}=6.64 \times 10^{-26} \mathrm{~kg}$.

\section{Table 4. Overview of Case Studies with the Base Case Marked $^{a}$}

$\begin{array}{cccccccc}\text { case } & \text { model } & c[\text { wt } \%] & \begin{array}{c}\text { G cont. } \\ (\mathrm{H} / \mathrm{L})\end{array} & \begin{array}{c}M_{\mathrm{w}} \\ {[\mathrm{kDa}]}\end{array} & f[-] & \text { annealing } & \begin{array}{r}t_{\text {sim }} \\ {[\mu \mathrm{s}]}\end{array} \\ 1 & \text { IM1 } & 0.5 & \mathrm{H} & 200 & 0.1 & \text { no } & 10 \\ 2 & \text { IM1 } & 0.5 & \mathrm{H} & 200 & 0.2 & \text { no } & 10 \\ 3 & \text { IM1 } & 0.5 & \mathrm{H} & 200 & 0.35 & \text { no } & 10 \\ 4 & \text { IM1 } & \mathbf{0 . 5} & \mathrm{H} & \mathbf{2 0 0} & \mathbf{0 . 5} & \text { no } & 10 \\ 5 & \text { IM1 } & 0.5 & \mathrm{H} & 200 & 1.0 & \text { no } & 10 \\ 6 & \text { IM1 } & 0.5 & \mathrm{~L} & 200 & 0.1 & \text { no } & 10 \\ 7 & \text { IM1 } & 0.5 & \mathrm{~L} & 200 & 0.2 & \text { no } & 10 \\ 8 & \text { IM1 } & 0.5 & \mathrm{~L} & 200 & 0.35 & \text { no } & 10 \\ 9 & \text { IM1 } & 0.5 & \mathrm{~L} & 200 & 0.5 & \text { no } & 10 \\ 10 & \text { IM1 } & 0.5 & \mathrm{~L} & 200 & 1.0 & \text { no } & 10 \\ 11 & \text { IM1 } & 0.5 & \mathrm{H} & 100 & 0.1 & \text { no } & 10 \\ 12 & \text { IM1 } & 0.5 & \mathrm{H} & 100 & 0.2 & \text { no } & 10 \\ 13 & \text { IM1 } & 0.5 & \mathrm{H} & 100 & 0.35 & \text { no } & 10 \\ 14 & \text { IM1 } & 0.5 & \mathrm{H} & 100 & 0.5 & \text { no } & 10 \\ 15 & \text { IM1 } & 0.5 & \mathrm{H} & 100 & 1.0 & \text { no } & 10 \\ 16 & \text { IM2 } & 0.5 & \mathrm{H} & 200 & 0.5 & \text { no } & 40 \\ 17 & \text { IM1 } & 0.5 & \mathrm{H} & 200 & 0.1 & \text { AN2 } & 15 \\ 18 & \text { IM1 } & 0.5 & \mathrm{H} & 200 & 0.2 & \text { AN2 } & 15 \\ 19 & \text { IM1 } & 0.5 & \mathrm{H} & 200 & 0.5 & \text { AN2 } & 20 \\ 20 & \text { IM1 } & 0.5 & \mathrm{H} & 200 & 1.0 & \text { AN2 } & 20 \\ 21 & \text { IM1 } & 0.5 & \mathrm{~L} & 200 & 0.5 & \text { AN2 } & 20 \\ 22 & \text { IM1 } & 0.5 & \mathrm{H} & 100 & 0.5 & \text { AN2 } & 20 \\ 23 & \text { IM2 } & 0.5 & \mathrm{H} & 200 & 0.5 & \text { AN2 } & 40 \\ 24 & \text { IM1 } & 1.0 & \mathrm{H} & 155 & 0.5 & \text { no } & 10\end{array}$

${ }^{a}$ All cases are in a $500 \mathrm{~nm}$ cubic box with periodic boundary conditions. 


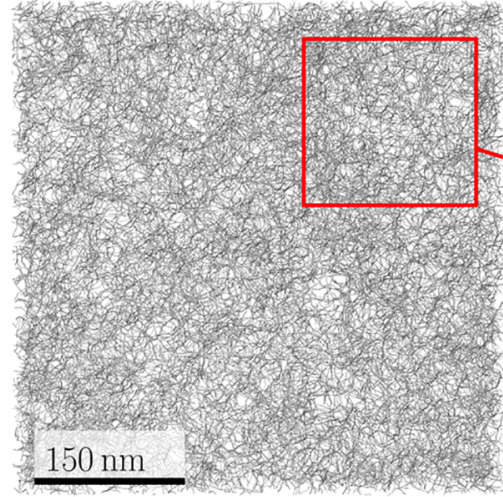

(a)

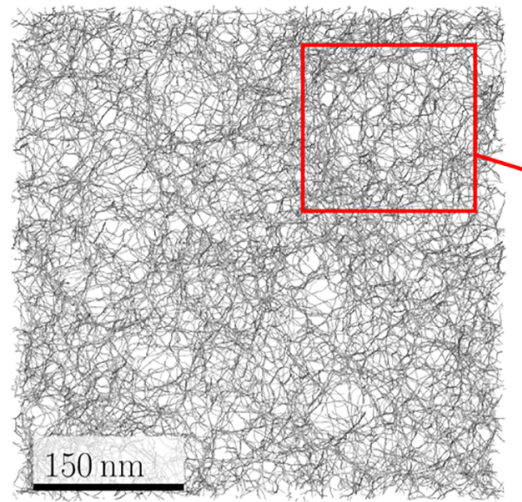

(c)

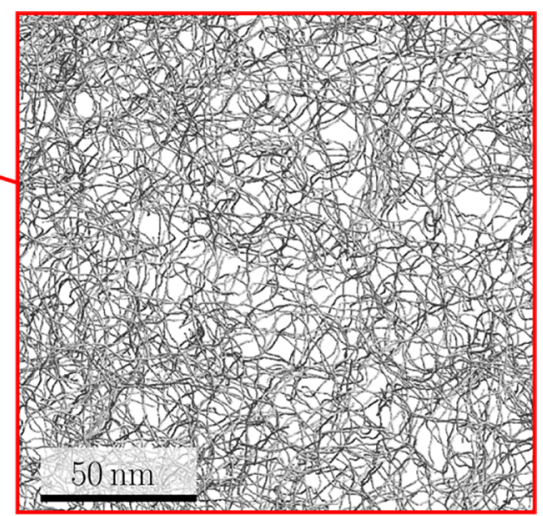

(b)

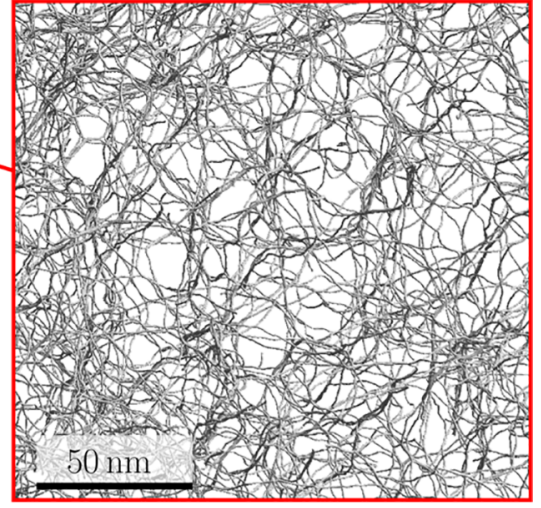

(d)

Figure 3. Comparison of nongelled polymer solution, i.e., without ions, (a) with a polymer gel of $f=0.5$ ions (c) using the ion-binding model (IM1), see base case 4 . A section of $200 \mathrm{~nm}$ depth at the center of the box is displayed and bonds are visualized with a diameter of $0.7 \mathrm{~nm}$. For (b,d), a magnification of three is used.

5.1.7. Simulated Annealing. As outlined in Section 3.4, simulated annealing procedures are employed to reduce overall simulation times to reach equilibrium and improve the probability of reaching the global potential minimum over remaining in a local potential minimum. Depending on the step of the simulation procedure, different annealing procedures and settings have been developed and wherever possible validated against runs without annealing. Typically used annealing procedures and settings are

1. AN1: Linear decay, $T_{\text {an, } \max }=2000 \mathrm{~K}, \tau_{\text {an, cool }}=5 \mu \mathrm{s}, \tau_{\text {an,period }}$ $=5 \mu \mathrm{s}$, and $t_{\text {an,finished }}=5 \mu \mathrm{s}$ (typically used for the relaxation step)

2. AN2: Linear decay, $T_{\mathrm{an}, \max }=2000 \mathrm{~K}, \tau_{\mathrm{an}, \mathrm{cool}}=2 \mu \mathrm{s}, \tau_{\text {an,period }}$ $=2 \mu \mathrm{s}$, and $t_{\mathrm{an}, \text { finished }}=10 \mu \mathrm{s}$ (typically used for the production step)

Validation of the annealing procedure did most notably take place for the relaxation step and was found to produce equivalent system properties in relation to the BAC. Concerning the annealing procedure for production runs, differences to simulations without annealing are discussed in the Simulation Results section.

5.2. Case Studies. A total of 24 case studies were simulated, and an overview can be found in Table 4 . All case studies were performed on a $0.5 \mu \mathrm{m}$ box (volume $0.125 \mu \mathrm{m}^{3}$ ) with periodic boundary conditions and primarily a constant polymer concentration $c$ of $0.5 \mathrm{wt} \%$ as well as a selected run at $1.0 \mathrm{wt}$ $\%$ for comparison with experimental data. The primary focus of the case studies was the investigation of the impact of calcium concentration $(f=0.1-1.0)$ using the binding model IM1 for systems with a high $\mathrm{G}$ content and a molecular weight of 200 $\mathrm{kDa}$. Furthermore, systems with low $\mathrm{G}$ content and a molecular weight of $100 \mathrm{kDa}$ were investigated, as well as a selected run at an intermediate value of $155 \mathrm{kDa}$. Additionally, studies were performed using the ion-unbinding model IM2 at a constant ion concentration of $f=0.5$, which were computationally significantly more expensive. For many systems in addition to a simulation without annealing, a simulation with annealing procedure AN2 was performed. Furthermore, equilibration results provided the reference without calcium $(f=0.0)$ for each case.

5.3. Simulation Results. 5.3.1. Base Case (4). The fully gelled case 4 was chosen as the base case for this study. The structural formation of the gel can be visually seen in Figure 3, where the thin layer with a depth of $200 \mathrm{~nm}$ is shown. An overview of the time-dependent quantitative results can be found in Figure 4 including all potential components (a), ion balance (b), average (c), and histogram (d) of the coordination number for a contact diameter of $0.9 \mathrm{~nm}$, normalized pairwise contacts (e) for an LJ cutoff of $1.5 \mathrm{~nm}$ if above $1 \%$ of all contacts, and volume fractions of dimer concentrations (f) based on a 5 $\mathrm{nm}$ voxel discretization.

As it can be seen in Figure 3a, the nongelled polymer solution is very homogeneous with individual fibers and fine spaces in between them. During gelation (see Figure $3 \mathrm{~b}$ ), bundle and network formation of polymer fibers can be observed. Approximately half of all particles/dimers take place in an interaction with other fibers (Figure $4 \mathrm{~d}$ ) and indicate a dense network formation. At the same time, pores on scales of $\mathrm{O}(10)$ 


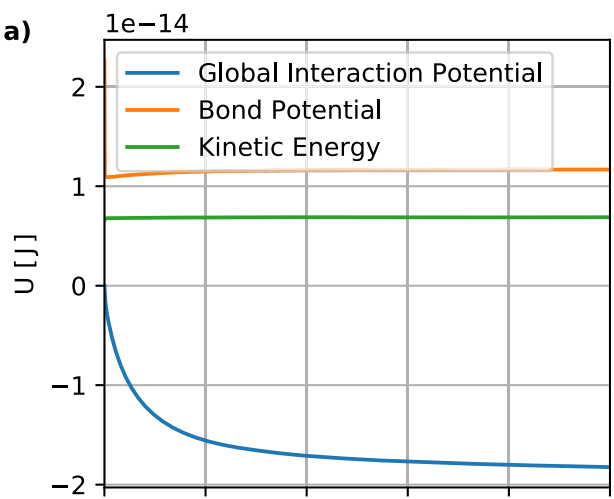

c)

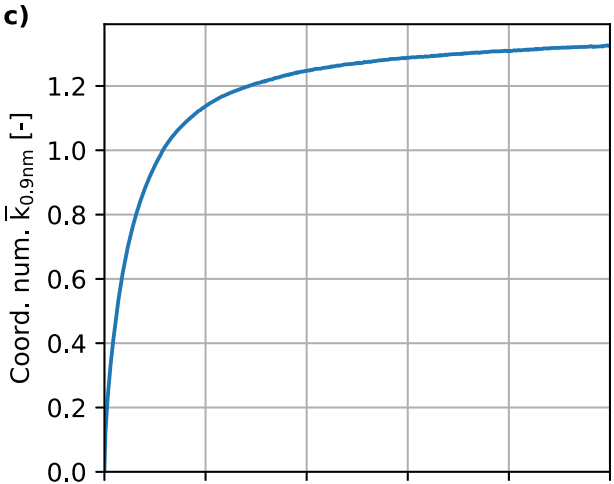

e)

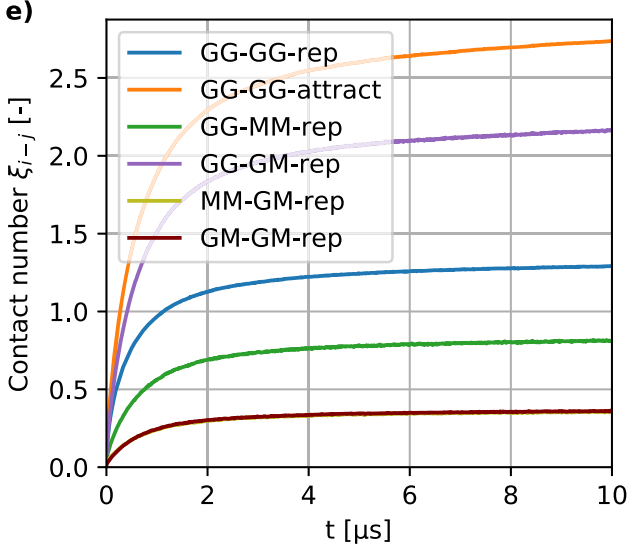

b)
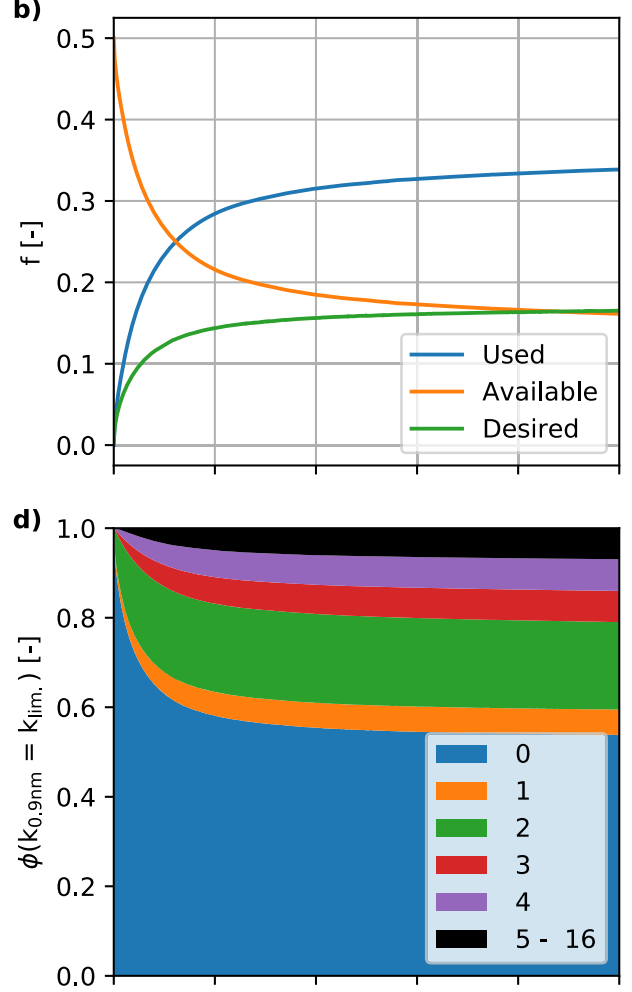

f)

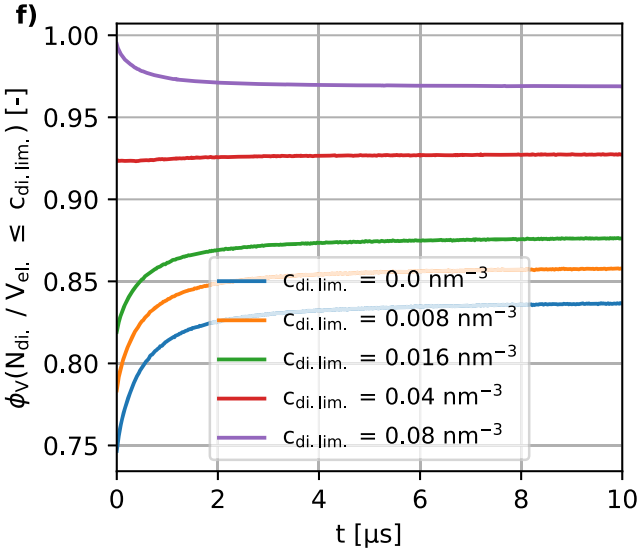

Figure 4. Overview of simulation results of the base case 4. See Section 3.6 for more details on the properties.

$\mathrm{nm}$ and above form. Due to the anisotropy and interconnectivity of these pores, as well as often crossing individual fibers, precise quantification is challenging. To address this, concentration volume fractions will be discussed in the following section on time-dependent data showing a significant increase in pore volume, as well as chord-length distributions in Supporting Information S1.3.

As it can be seen in Figure 4, the system reaches the steady state in approximately $4 \mu \mathrm{s}$. At this point, all system properties near their asymptotic values with respect to its fast modes of relaxation and the kinetics significantly slow down. From the obtained results, it can be observed that only $f=0.34$ ions are used by the polymer during gelation and the remaining $f=0.16$ ions remain available. At the same time, $f=0.17$ ions remain desired by pairwise interactions, that is, representing unbonded contacts capable of receiving an ion. This is attributed to the zipping mechanism of polymer chains and the (modeled) inability to accept ions once the dimers are in close proximity. These pairwise interactions desiring an ion are likely going to receive one if available due to a thermodynamically driven opening of the pairwise interaction in both the physical and modeled system. However, the time scales of such a process are likely much larger than possible to be simulated. Additionally, the simplified model likely deviates from the physical systems to some extent in this regard.

Furthermore, the network formation during gelation can be observed in Figure 4c,d, which shows the coordination number. Initially, almost no connections exist between dimer units besides neighboring ones on the same polymer chain. Over time, the coordination number increases to an average of 1.3 with more than $46 \%$ of dimers having a coordination number of at least one. As it can be seen in Figure $4 d$, the coordination number goes up to 16 in rare cases, which is attributed to the inside of polymer bundles. However, 93\% remain below a coordination number of 5 and $99.98 \%$ below 10 . Additionally, as it can be seen in Figure 4e, each GG dimer enters on average 2.74 attractive egg-box contacts, indicating polymer bundle 
formation, which is in agreement with experimental expectations.

Lastly, Figure $4 \mathrm{f}$ shows the volume fraction below specific concentration limits. As it can be seen, the volume fraction of empty cells increases with gelation from 0.75 to 0.84 , while the fraction of cells with a concentration higher than $0.08 \mathrm{~nm}^{-3}$ (i.e., 10 dimers per $5 \mathrm{~nm}$ voxel) increases from 0.005 to 0.03 . This trend of an increase in pore volume together with an increase in bundle volume is in good agreement with expected trends during gelation.

Overall, it can be said that the presented simulation results likely present the ideally homogeneous case. Experimental systems will likely contain more inhomogeneities due to processing (e.g., shear) and composition (e.g., impurities), leading to larger polymer bundles and pores.

5.3.2. Case Studies. In the following section, the results of all case studies will be present split into those without annealing and those with annealing procedure AN2. For this, the properties described in Section 3.6 at the end of each simulation run will be compared, and visualizations provided. The overview of all results can be found in Figures 5 and 6 for no annealing and in Figures 7 and 8 with annealing procedure AN2.
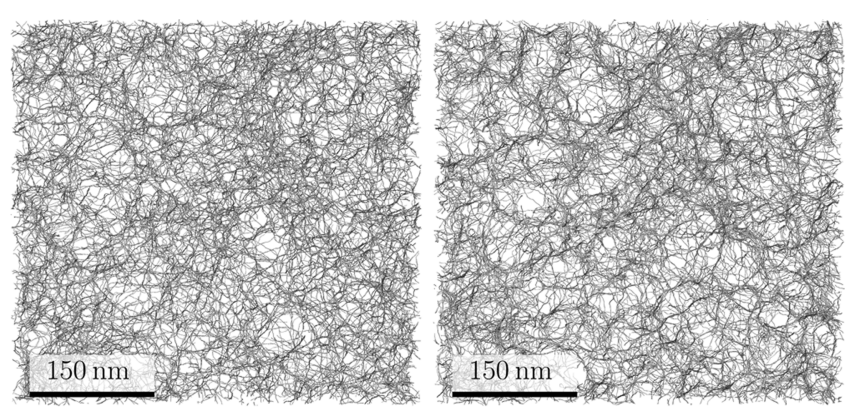

(a) Case 4: IM1, H, $200 \mathrm{kDa}$.

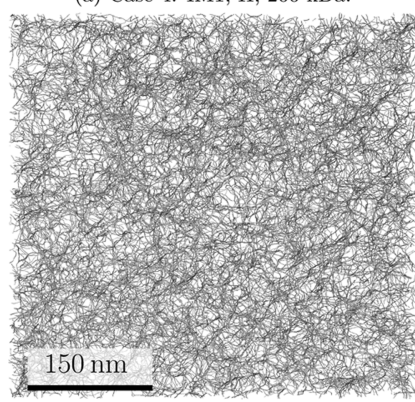

(c) Case 9: IM1, L, $200 \mathrm{kDa}$. (b) Case 16: IM2, H, $200 \mathrm{kDa}$.

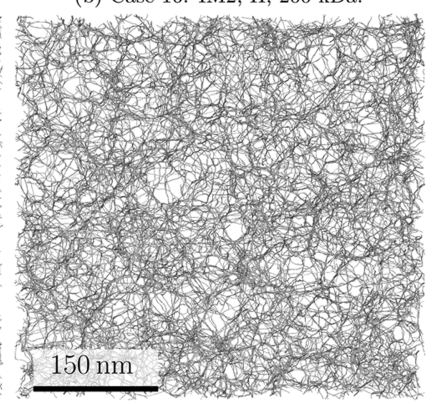

(d) Case 14: IM1, H, $100 \mathrm{kDa}$.
Figure 5. Visual comparison of cases without annealing for a polymer concentration of $0.5 \mathrm{wt} \%$ and an ion concentration of $f=0.5$. A section of $200 \mathrm{~nm}$ depth at the center of the box is displayed, and bonds are visualized with a diameter of $0.7 \mathrm{~nm}$.

5.3.2.1. Constant Temperature-No Annealing. The case studies without annealing present the lower limit of polymer network formation during gelation, as these simulations are more prone to be limited by local potential minima and consequently limited equilibration for the time scales possible to be addressed using simulations. In this regard, they are very important to understand the mechanics of gelation and its modeling. A visualization of the gelled systems using constant temperature can be found in Figure 5. The overview of all quantitative results of simulations without annealing can be found in Figure 6.

Before going into the quantitative analysis, the visualizations in Figure 5 at a constant ion concentration of $f=0.5$ provide a qualitative overview of the impact of other system parameters. As it can be seen, the comparison of the binding model IM1 (a) and unbinding model IM2 (b), both provide similar gel networks with a slight tendency toward thicker polymer bundles and visually slightly larger pores for the unbinding model IM2. Concerning the impact of fiber composition (c), a lower G content leads to a decrease in bundle thickness and pore sizes. As we discuss later in Section 6, it is only clear how the bundle size is influenced by the degree of cross-linking $f$, but the role of other factors such as molecular weight and G content has never been addressed in the literature, to the best of our knowledge. Therefore, the predictions made in this study lay a basis for further experimental studies. Concerning the impact of molecular weight (d), a decrease in molecular weights to 100 $\mathrm{kDa}$ leads to an increase in bundle thickness and pore sizes.

In the following, we first focus on the $200 \mathrm{kDa}$ and high $\mathrm{G}$ content system (see Figure 5a and blue data in Figure 6). As it can be seen for all parameters in Figure 6, an asymptotic behavior with regard to ion concentration can be observed. Asymptotic values of all parameters reached around $f=0.35$, which are further supported by the concentration of used ions (see Figure $6 \mathrm{~b}$ ) between $f_{\text {used }}=0.34-0.38$ for $f=0.5$ and 1.0, respectively. At this point, the system appears to be saturatedalthough as was discussed in Section 5.3.1, closed off interaction zones still desire ions, which might be accepted by those interactions over a longer period of time. However, this is not expected to actually change the gel structure. Overall, the discussion of the base case in Section 5.3.1 consequently holds true for the asymptotic case. Additionally, these findings can be used to estimate the critical ion concentration for gelation and compared to experimental findings. The functional behavior of all system properties is approximately exponential and half of the asymptotic value is reached at approximately $f=0.2$. Consequently, this value could be used as a rough estimate of the critical ion concentration for gelation. We found that data by Lu et al. ${ }^{69}$ on the critical cross-linking degree $f_{\text {crit }}$ is a linear function of $c_{\text {alg }}$ and extrapolated to $0.5 \mathrm{wt} \%$ concentration. This yielded for critical $f$ an estimate of 0.17 , in excellent agreement with our estimation.

Next, the impact of changing to a low G content system keeping the molecular weight constant at $200 \mathrm{kDa}$ will be discussed (see Figure 5c and green data in Figure 6). As it can be observed in Figure $6 \mathrm{~d}$, the asymptotic contact number $\xi_{\mathrm{GG}-\mathrm{GG}-\text { attract }}$ is approximately the same as the high $\mathrm{G}$ system, indicating that the normalized egg-box formation remains indifferent of $\mathrm{G}$ content. However, the overall gel structure appears to be not as densely interconnected, as indicated by the lower coordination number (c) and reduced pore volume (f), but also has the increased global interaction potential (a) and reduced used ion concentration (b). This behavior is expected and attributed to the fact that the formation of GG-GG connections (egg-box) is crucial for the gel formation, and consequently, a lower $\mathrm{G}$ composition is expected to lead to a less densely connected gel. Additionally, the data indicate that the asymptotic gelation is reached at lower ion concentrations of approximately $f=0.2$ as also supported by $f_{\text {used }}=0.15$ at $f=0.5$. Fang et al. ${ }^{36}$ have found that egg-box dimers start to form when the ratio $\left[\mathrm{Ca}^{2+}\right] /[\mathrm{G}]$ is in the range $0.25-0.55$. This interval thus can be considered as a critical cross-linking degree. In our 


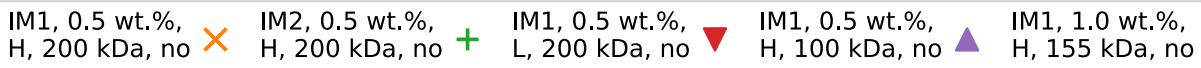

a)

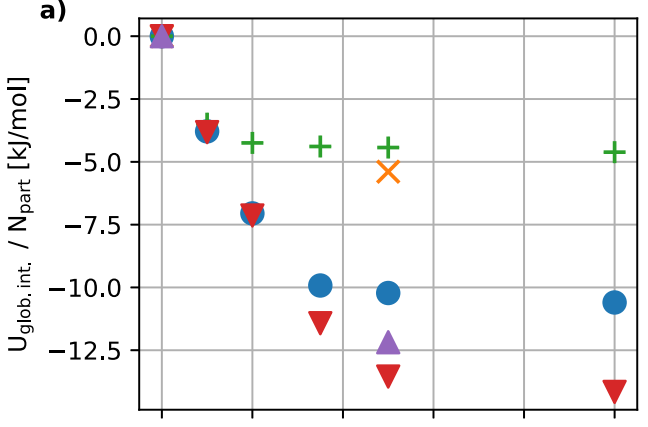

c)

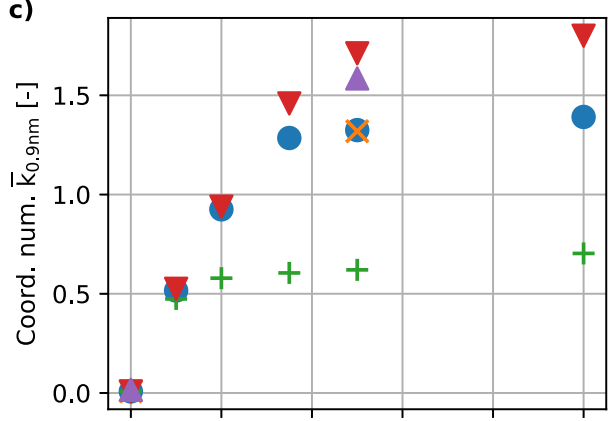

e)
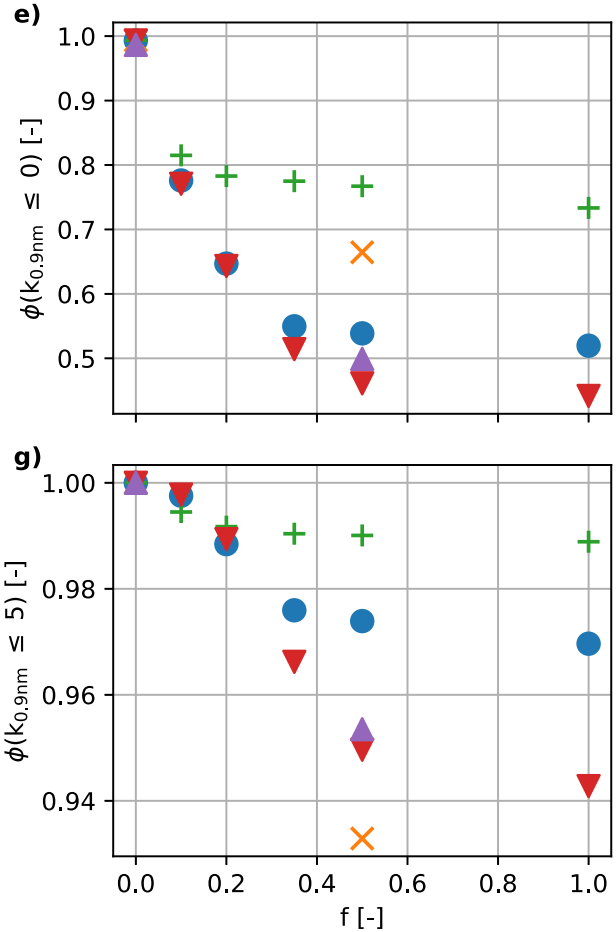

b)

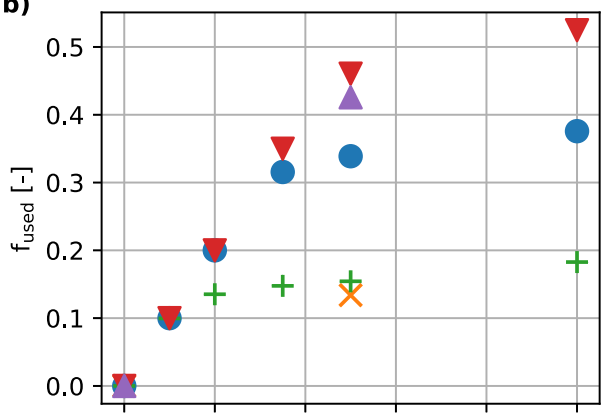

d)

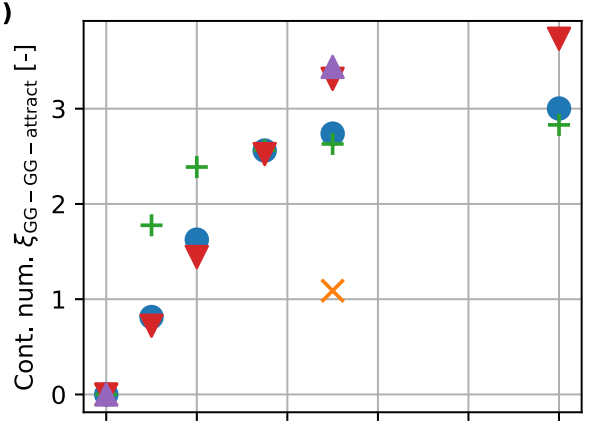

f)

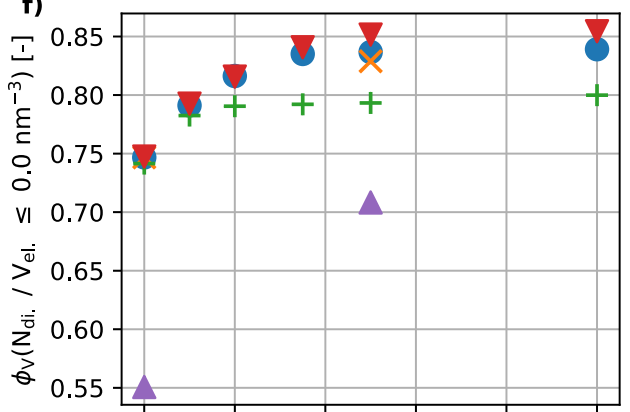

h)

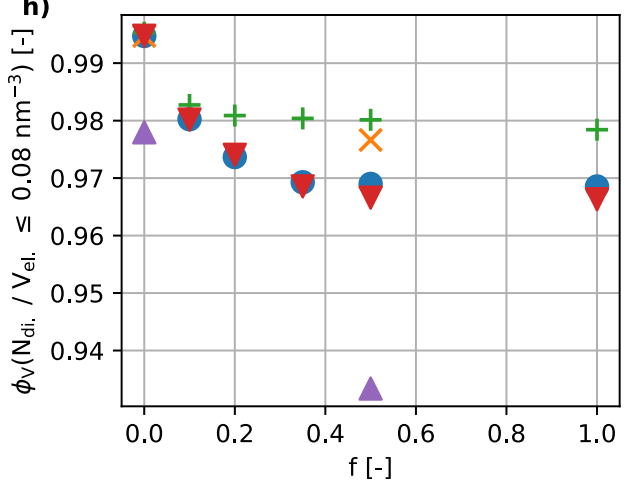

Figure 6. Overview of simulation results of all case studies without annealing.

terms, the cross-linking degree $f$ should reach $0.1-0.2$ for asymptotic gelation to be observed, which is in excellent agreement with our estimation.

Next, the impact of changing the molecular weight to 100 kDa keeping the high $\mathrm{G}$ content will be discussed (see Figure $5 \mathrm{~d}$ and red data in Figure 6). The data indicate that the reduced molecular weight causes a more densely connected gel network with increased polymer bundle thickness (see increased fraction of the coordination number above 5 in Figure $6 \mathrm{~g}$ ), as well as slightly increased pore volume (see Figure 6f). Additionally, the lower molecular weight uses up significantly more ions, leading to a lower global interaction potential, as further supported by the increased normalized GG-GG attractive connections. This influence of lower molecular weight is attributed to the increased mobility of polymer fibers. As the polymer fibers are essentially only half as long and consequently not as constrained due to cross-linkage, bundle formation is supported, which also leads to a higher pore volume.

Next, the impact of changing to a higher polymer concentration of $1 \mathrm{wt} \%$ and intermediate molecular weight 

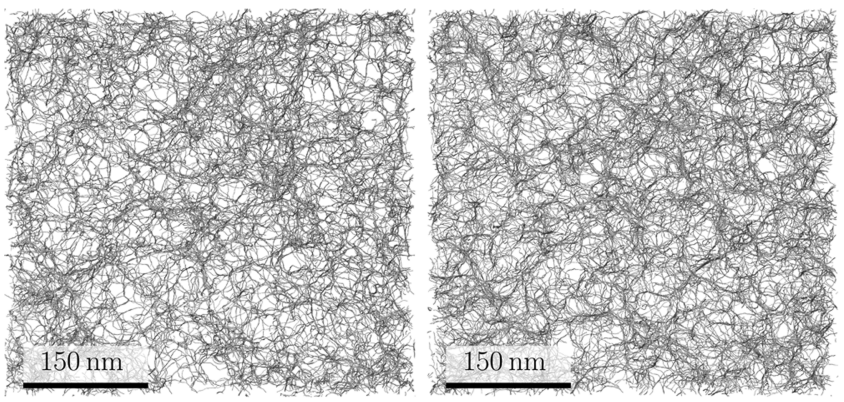

(a) Case 19: IM1, H, $200 \mathrm{kDa}, \mathrm{AN} 2$.

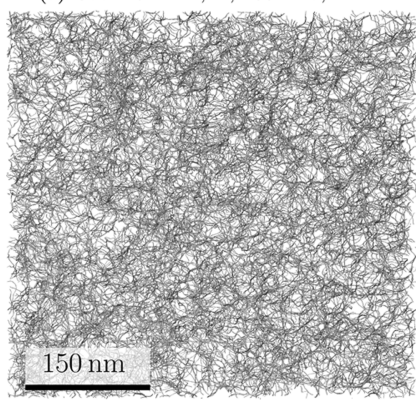

(c) Case 21: IM1, L, $200 \mathrm{kDa}, \mathrm{AN} 2$ (b) Case 23: IM2, H, $200 \mathrm{kDa}, \mathrm{AN} 2$.

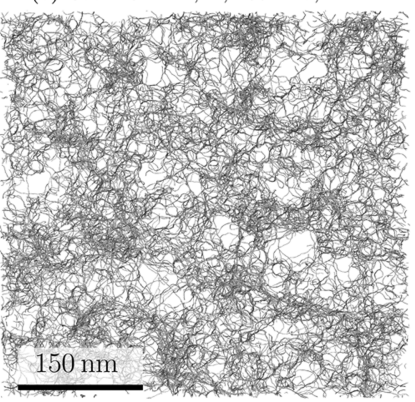

(d) Case 22: IM1, H, $100 \mathrm{kDa}, \mathrm{AN} 2$.

Figure 7. Visual comparison of cases with annealing procedure AN2 for a polymer concentration of $0.5 \mathrm{wt} \%$ and an ion concentration of $f=$ 0.5 . A section of $200 \mathrm{~nm}$ depth at the center of the box is displayed and particles are visualized with a diameter of $0.7 \mathrm{~nm}$.

of $155 \mathrm{kDa}$, while keeping the high $\mathrm{G}$ content, will be discussed (violet data in Figure 6). The data indicate a densely connected gel network with an intermediate bundle thickness between the two molecular weights (100 and $200 \mathrm{kDa}$ ) at $0.5 \mathrm{wt} \%$. Consequently, the impact of the molecular weight appears to be dominating in regard to bundle thickness as it can be seen in the intermediate average coordinate number in Figure 6c, intermediate fraction of coordination number of zero in Figure $6 \mathrm{e}$, intermediate global interaction potential in Figure 6a, and intermediate fraction of used ions in Figure $6 \mathrm{~b}$. Additionally, as the increased coordination number of attractive GG-GG "eggbox" interactions in Figure $6 \mathrm{~d}$ and the coordination number above five in Figure $6 \mathrm{~g}$ indicate, further effects on bundle thickness can also be attributed to the increase in polymer concentration. However, the largest effects of the increased polymer concentrations are with regard to the significantly decreased overall pore volume (Figure 6f) and increased bundle volume (Figure $6 \mathrm{~h}$ ). These effects are consistent with expectation resulting from higher concentrations leading to a higher occupied volume by the polymer fibers. Further discussions will be provided in context with the comparison to experimental data in Section 6.

Next, the impact of the ion-unbinding model IM2 will be discussed (see Figure $5 \mathrm{~b}$ and orange data in Figure 6). This model describes the loss of an ion bound to 1-2 dimers (i.e., particles) during interaction and consequently attempts to model the dynamic equilibrium of ion binding and unbinding. It was found that the time to reach the steady state increases by a factor of 2-4 (AN2-no annealing), leading to a 2-4× increase in computational requirements (17 days for $40 \mu$ s on a Nvidia A100 GPU). Consequently, only two data points could be simulated (no annealing/AN2), which were performed at $f=0.5$. As Figure 6 shows, the average coordination number in (c) and

pore fraction in (f) are almost identical, indicating similar gels. However, as it can be seen in (d), approximately half of the GGGG interactions (egg-boxes) appear to have lost their ion during polymer bundle formation/zipping, leading additionally to only half as many used ions and half the global interaction potential. Consequently, many bundles appear to have egg-boxes without an ion. Additionally, as indicated by the increase in the fraction of particles with a coordination number of zero in (e), this appears to reduce cross-linkage of individual polymer fibers. This in turn then supports the formation of thicker polymer bundles as indicated by the increased particle fraction of coordination numbers above 5 in $(\mathrm{g})$ (similar to a reduction of molecular weight). However, these thick bundles appear to fill a smaller volume fraction as indicated in $(\mathrm{h})$. Overall, it can be said that the ion-unbinding model IM2 produces slightly different gels. The results indicate thicker polymer bundles, but overall, the same pore volume and coordination number, that is, specific surface. As experimental validation, especially in this idealized case, remains challenging to this date, it is difficult to assess which model is more appropriate and will likely require further research and a more detailed investigation of ion binding/ unbinding. Consequently, in light of computational requirements and the ability to reproduce experimentally/empirically reasonable trends, the simple binding model presents the prudent choice at this point. However, this assessment might change at some point later depending on additional and/or supporting data concerning the ion binding/unbinding.

5.3.2.2. Annealing Procedure AN2. In contrast to the simulation studies without annealing, which represent the lower limit of gelation, the studies with annealing procedure AN2 attempt to resolve many of the limitations of local potential minima. However, these improvements come at the cost of increased computational requirements, as well as the possibility of artifacts. A visualization of the gelled systems using annealing procedure AN2 can be found in Figure 7. The overview of difference in simulation results between no annealing and AN2 can be found in Figure 8 .

Before going into the quantitative analysis, the visualizations of Figure 7 in comparison with Figure 5 provide a qualitative overview of the impact of the annealing procedure at an ion concentration of $f=0.5$. Visually, the unbinding model IM2 (b) and low $\mathrm{G}$ content system (c) provide very similar results, while the base case (a) and low-molecular-weight case (d) show slightly larger bundles and pore sizes. The low-molecular-weight case (d) also appears to be more disorganized near the junction zones, indicating possible artifacts. In the following, these differences will be quantified and discussed in relation to the ion concentration.

As it can be seen in Figure 8, for a low ion concentration $(f \leq$ 0.2 ), there is no significant difference between the results with AN2 and without annealing. These results are reasonable, as at this point, few energy barriers are expected due to the reduced gelation. Additionally, even at $f=0.5$, the results for the systems with a low $G$ content and unbinding model (IM2) remain similar to constant temperature (no annealing), while the base case (IM1, H, $200 \mathrm{kDa}$ ) and $M_{\mathrm{w}}=100 \mathrm{kDa}$ case show higher differences. Concerning the low G content system, this is reasonable, as the energy barriers are much lower in comparison with the high $\mathrm{G}$ content system due to the reduced number of GG units, that is, leading to fewer stable egg-box interactions potentially acting as an energy barrier. Concerning the unbinding model (IM2), this result is expected, as the unbinding probability leads to a similar effect as the annealing procedure. 

IM1, 0.5 wt. \%,
$\mathrm{H}, 200$ kDa, AN2-no $X$

IM2, 0.5 wt. \%, $\mathrm{H}, 200 \mathrm{kDa}, \mathrm{AN} 2-\mathrm{no}+$
IM1, 0.5 wt. \%, L, 200 kDa, AN2-no
IM1, 0.5 wt. \%, $\mathrm{H}, 100 \mathrm{kDa}$, AN2-no

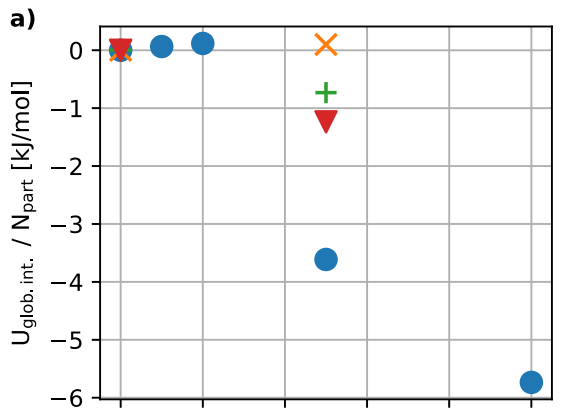

c)

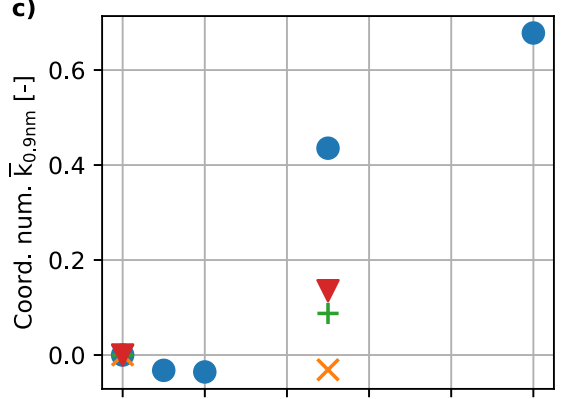

e)
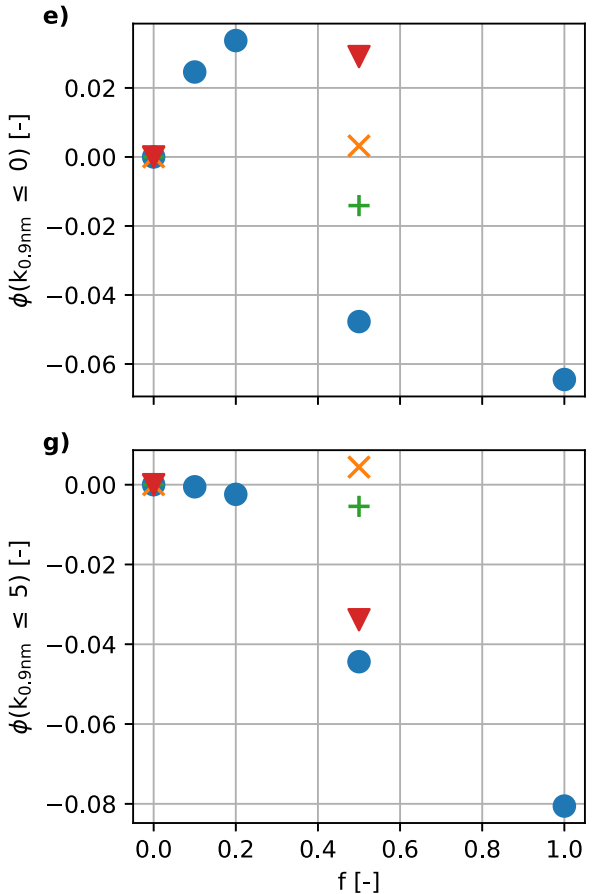

b)
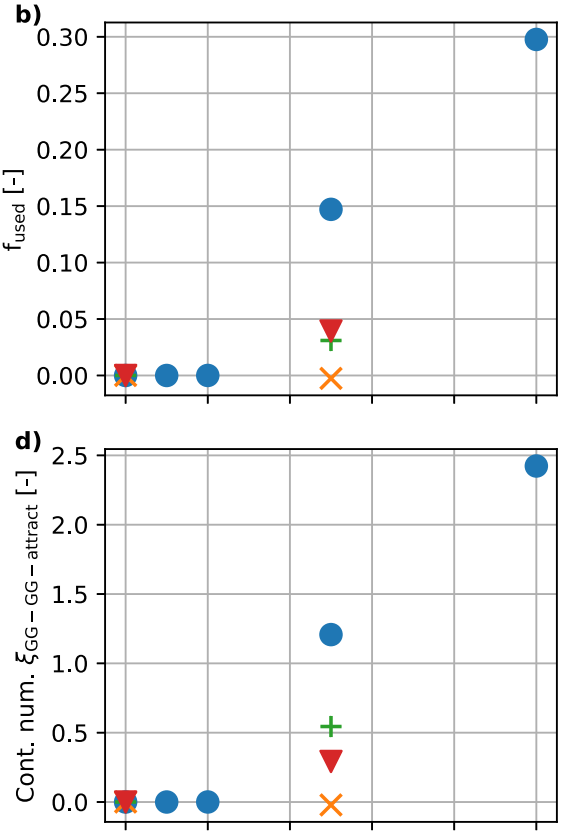

f)

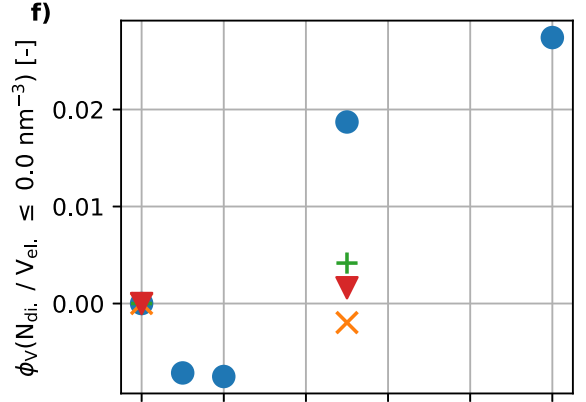

h)

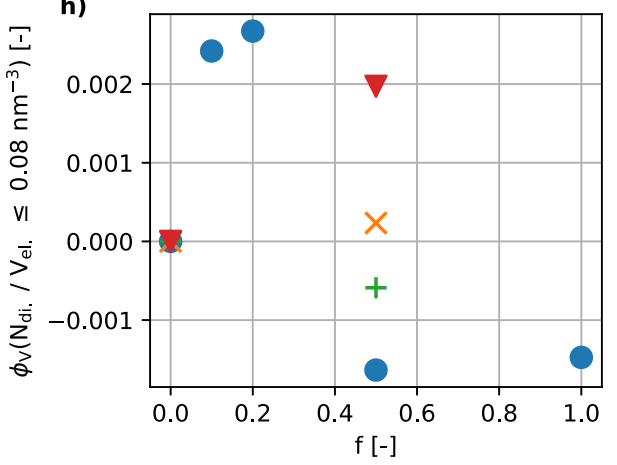

Figure 8. Overview of the difference in simulation results between annealing procedure AN2 and no annealing (AN2-no).

Concerning the system with a reduced molecular weight of $100 \mathrm{kDa}$, the differences remain significantly lower than that of the base case. As the main difference between this case and the base case is primarily a reduced cross-linkage of individual/few polymers over bundle formation (see previous section), the main differences of annealing might be attributed to this.

Overall, the annealing procedure AN2 has an effect primarily on systems with a high $\mathrm{G}$ content, high molecular weight (lower influence), and high ion concentration using the binding model (IM1). Due to the temporary breakage of interactions, it then leads to a higher acceptance of ions and consequently a higher coordination number (Figure 8c) and egg-box formation (GG-
GG attractive interaction, Figure $8 \mathrm{~d}$ ). As a result, the fraction of single fibers reduced (Figure 8e), the fraction of thick bundles increased (Figure $8 \mathrm{~g}$ ), and the pore volume fraction increased (Figure 8f).

In conclusion, the annealing procedure further supports gelation and leads to increased bundle sizes and pore volume in some cases [high G content and high molecular weight with lower influence for high ion concentrations using the binding model (IM1)]. Based on these results of a limited impact on other systems, limited impact on gelation trends, and doubling the computational requirements, the annealing procedure in production runs can only be partially recommended. 


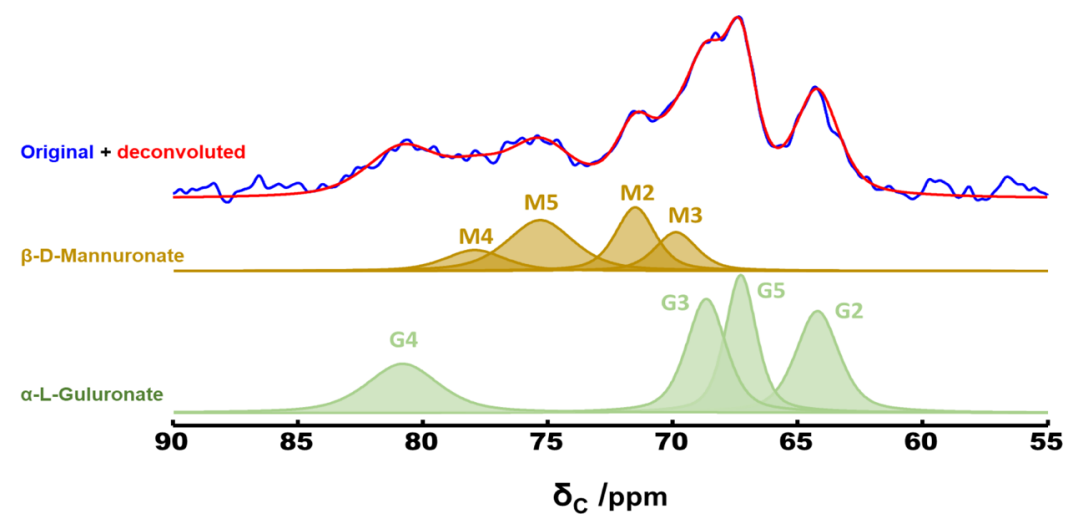

Figure 9. ${ }^{13} \mathrm{C}$ CPMAS NMR spectrum of the alginate hydrogel ( 1 wt \% gelled in $0.5 \mathrm{wt} \% \mathrm{CaCl}_{2}$ ) recorded with a MAS rate of $5 \mathrm{kHz}$ and a $\mathrm{CP}$ contact time of $10 \mathrm{~ms}$. The line shape is fitted to Gaussian and Lorentzian line shapes to deconvolute the overlapping peaks. The estimated $\mathrm{G}$ content is $0.66 \pm$ 0.10 .
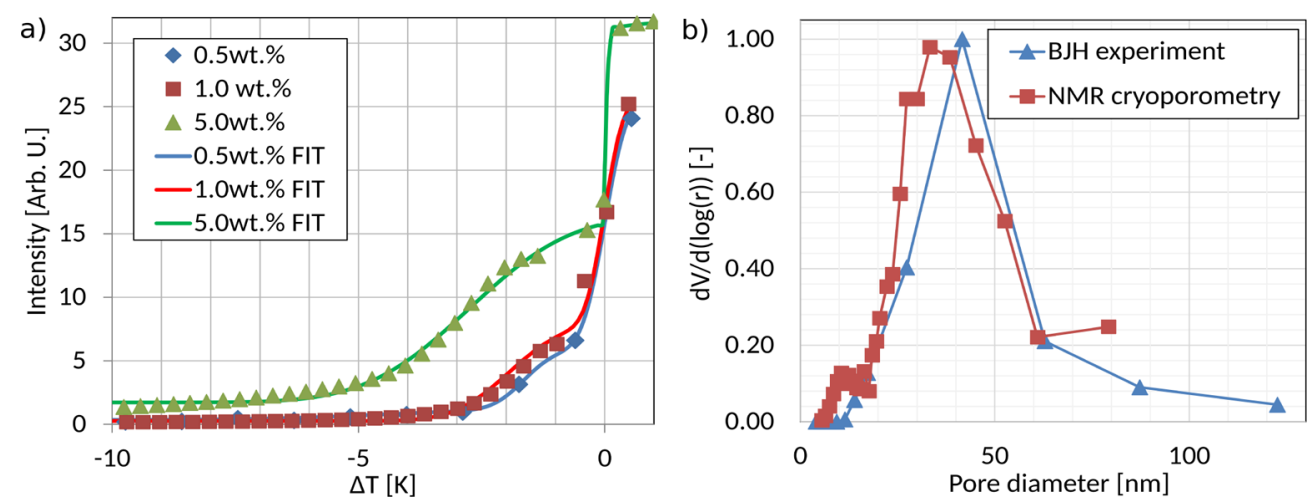

Figure 10. (a) NMR cryoporometry measurements of different Ca-alginate hydrogels. Water peak integrals in freezing experiments plotted against temperature calculated as a difference $(\Delta T)$ from the freezing point of the bulk liquid phase $(\Delta T=0)$. Markers: experimental data points. Continuous lines: fitted curves using multiple summarized error functions. (b) Averaged and normalized PSDs from NMR cryoporometry of hydrogels and nitrogen desorption of the corresponding aerogels. For raw data, see the Supporting Information.

\section{COMPARISON WITH OWN AND PUBLISHED DATA}

Sodium alginate used in this study was the same as "high-G" alginate from Agulhon et al. ${ }^{66}$ and their previous works where the content of $\mathrm{G}$ and $\mathrm{M}$ units was reported to be 0.63 and 0.37 , respectively (without uncertainty estimation). We independently measured (Figure 9, also see Supporting Information S1.4) the G-content for hydrogels gelled in 0.5 and 1.0 wt $\% \mathrm{CaCl}_{2}$ to be $0.66 \pm 0.10$ and $0.70 \pm 0.10$, respectively, by ${ }^{13} \mathrm{C}$ CPMAS NMR. To maintain consistency with the previously published works, we accept the $G$ content value of 0.63 in our modeling.

Two sources of experimental data for model validation are employed in this work. First, we prepared three hydrogels crosslinked in different $\mathrm{CaCl}_{2}$ concentrations, $0.5,1.0$, and 5.0 wt $\%$. The calcium content was found to be $81.6 \pm 1.8,91.1 \pm 2.1$, and $85.0 \pm 0.90 \mathrm{~g} \mathrm{Ca} / \mathrm{kg}$-aerogel, respectively. The use of lower $\mathrm{CaCl}_{2}$ concentrations is impractical as it would slow down the gelation rate and would result in easily breakable hydrogels. Therefore, there are no significant differences in the calcium content across the hydrogels. The mean value for all compositions is $85.9 \pm 4.4$. This value corresponds to the cross-linking degree $f=0.38 \pm 0.02$, which is in reasonable agreement with simulation predictions $\left(f_{\text {used }}=0.43\right)$.

Calcium cross-linked alginate hydrogels were studied using NMR cryoporometry and SANS to quantify the PSD. Furthermore, hydrogels were converted into aerogels through a solvent exchange and supercritical drying. This procedure was shown to minimally influence the pore network. ${ }^{70}$ Thus, aerogels can be seen as solid replica of the parent hydrogels. The aerogels were analyzed with a nitrogen adsorptiondesorption method to obtain the PSD.

The NMR signal intensity in a melting-freezing experiment plotted as a function of $\Delta T$ yielded sigmoid curves in the case of all hydrogel samples, which is typical for porous materials. The freezing curve of each sample shows clear plateaus between $\Delta T$ $=-4$ and $0 \mathrm{~K}$, which is very advantageous for precisely estimating PSD curves from NMR cryoporometry data (Figure 10a).

The considerations of Petrov and Furo ${ }^{55}$ regarding pore geometry were taken into account when estimating pore sizes. The freezing curve of the Ca-alginate hydrogel of $5.0 \mathrm{wt} \%$ is shifted toward considerably lower $\Delta T$ than those of the other samples. This can indicate either significantly lower pore sizes or the change in the dominant pore geometry. Since other characterization methods do not indicate a significant difference between the pore sizes of the three different Ca-alginate hydrogels, we propose that the pore geometry of the $5.0 \mathrm{wt} \%$ sample is dominantly cylindrical, while the dominant pore geometry of the 0.5 and $1 \mathrm{wt} \%$ sample is intermediate between cylindrical and slab geometries. Thus, the form factor $n_{\mathrm{f}}=2$ was used for estimating the pore sizes of the $5.0 \mathrm{wt} \%$ sample, and $n_{\mathrm{f}}=$ 1 was used in the case of the other two hydrogels. Note that due to the anisotropic and interconnected nature of pores (void spaces among the loosely interweaved fibrillar structural elements), geometrical models are inherently challenging to 
be well-adapted to the Ca-alginate hydrogels and aerogels. The constant of $K_{c}=30 \mathrm{~nm} \mathrm{~K}$ was used in every case. ${ }^{55}$ Complete PSD curves for the hydrogels estimated from NMR cryoporometry data are given in the Supporting Information. It should be pointed out that the differences in the obtained PSD are rather minor. Analysis of the minor differences goes beyond the scope of the present study. Given that all samples have approximately the same degree of cross-linking (i.e., the same amount of calcium), it is sufficient for our purposes to average the PSD curves. The result of the averaging is shown in Figure $10 \mathrm{~b}$. Hydrogels, regardless of the calcium concentration in the gelation bath, possess a pore diameter of 30-36 nm. PSD curves were also measured for aerogels by $\mathrm{N}_{2}$ porosimetry $(\mathrm{BJH}$ method). They are also very similar across the materials studied (for raw data, see the Supporting Information) and were averaged. The data are shown in Figure $10 \mathrm{~b}$ along with the NMR cryoporometry results. The PSD curves of the aerogels are shifted to somewhat larger values ( $c a .42 \mathrm{~nm}$ ), which could be due to structural rearrangements because of supercritical drying. ${ }^{70,71}$ In addition, the uncertainty of the $K_{c}$ value $^{55}$ used in the calculation could also cause minor differences. The overall agreement between these two methods is very good. It can be therefore concluded that the most probable value of the pore diameter for the Ca-cross-linked hydrogels lies in the range 33$42 \mathrm{~nm}$.

The experimental SANS scattering curve of the Ca-alginate hydrogel is shown in Figure 11. The scattering curve was fitted

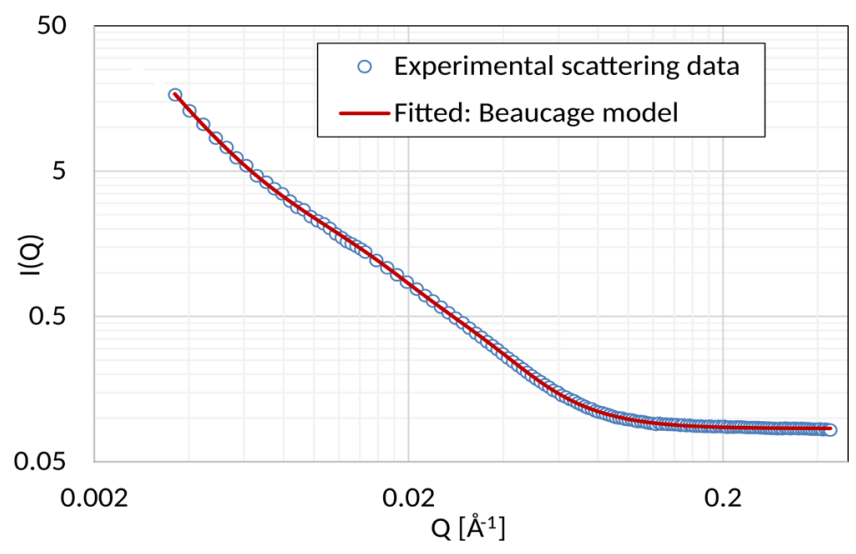

Figure 11. SANS of the Ca-alginate hydrogel in $\mathrm{D}_{2} \mathrm{O}$. Markers: experimental scattering curve. Continuous line: fitted curved based on the Beaucage model.

with different mathematical model functions including the ones based on elongated cylinders (with disperse lengths and radii). ${ }^{72}$ Unfortunately, none of the geometrical models describes the experimental scattering curve adequately. Finally, the Beaucage model gave an excellent fit in the complete experimental $Q$ range. Two Porod regions were identified with estimated powerlaw constants of $p_{1}=2.78 \pm 0.03$ and $p_{2}=3.04 \pm 0.02$. Both of these are characteristic for mass fractal-type scattering objects. The estimated gyration radius is $R_{\mathrm{g}}=10.4 \pm 0.1 \mathrm{~nm}$. Based on our previous experience with hydrated Ca-alginate gels, the gyration radius is assumed to represent the average distance between the Ca-alginate fibers (bundles) in the hydrogel, which in turn reflects the mean pore size. ${ }^{51}$ If the irregularly shaped pores are approximated with elongated cylinders, the pore radius can be calculated as $r_{\text {pore }}^{2}=2 R_{\mathrm{g}}^{2}$. 61 This rough approximation yields $29.4 \pm 0.3 \mathrm{~nm}$ for the mean pore diameter.
The pore diameter for the pores in the mesoporous range (2$50 \mathrm{~nm}$ ) determines the specific surface area of an aerogel, its key property. Therefore, it is of interest to be able to predict the average pore diameter and the entire PSD. A survey across several publications on alginate aerogels reveals that the pore diameter lies in a broad range with a median value of $23 \mathrm{~nm}$ and an interquartile range of $17 \mathrm{~nm}(n=7$, see Supporting Information S1.5). Hence, the values of the pore diameter obtained in the present study are rather at the higher end. As we mentioned in the introduction, there is no clear understanding on what factors influence the textural properties of hydro- and aerogels. Moreover, complete characterization of alginate and careful documentation of the process conditions (most importantly the calcium content) are missing in most of the papers on the topic. In such a situation, it is impossible to compare the model with the literature data.

By comparing the PSD curves from Figures $10 \mathrm{~b}$ and 12 (see Section 3.6 for postprocessing procedure), it can be seen that

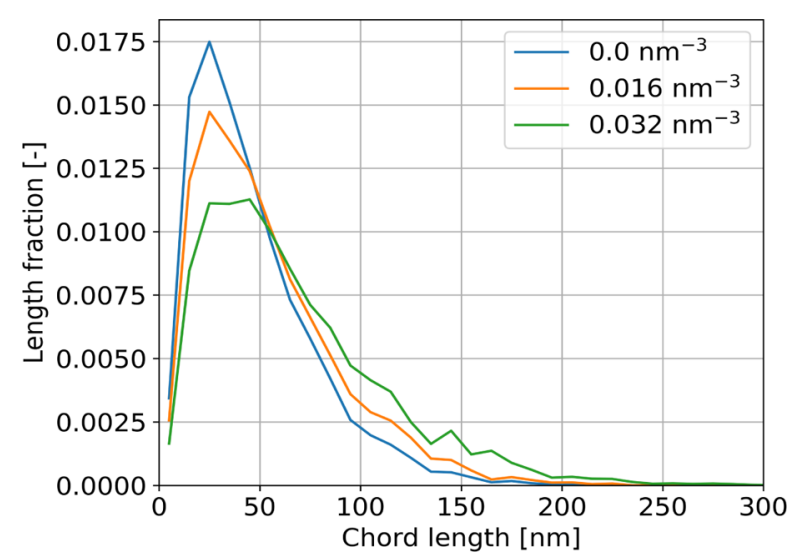

Figure 12. Numerical pore size through chord length distribution of simulation at $c=1 \mathrm{wt} \%, M_{\mathrm{w}}=155 \mathrm{kDa}$, high $\mathrm{G}$ composition, $f=0.5$, using IM1. Various curves indicate limit concentration under which a cell is considered empty, i.e., belonging to a pore.

simulation results indicate slightly smaller pore sizes in comparison with experimental results but generally agree with respect to pore sizes. As it can be seen in Figure 12, depending on the exact definition of a pore (i.e., a $5 \mathrm{~nm} \times 5 \mathrm{~nm} \times 5 \mathrm{~nm}$ voxel occupied by a single dimer considered a pore or not), the most probable pore size varies between 20 and $40 \mathrm{~nm}$. Results of nitrogen sorption experiments (BJH), NMR cryoporometry, and SANS all lie within this range (Figure 13). Given very different physical basis of the experimental techniques as well possible inhomogeneities within the sample, this agreement is very reasonable.

The second structural feature, extensively reported in the literature for alginate hydrogels, is the characteristic size of fibrils/bundles. In Table 5, we provide a summary of SAXS measurements for Ca-cross-linked hydrogels and aerogels. The reported fibril diameters vary by up to 1 order of magnitude depending on alginate concentration, alginate composition, molecular weight, ion concentration, and processing conditions. In this context of modeling validation, the most similar data will be used in the following.

The following conclusions can be drawn from the data in Table 5. First, the characteristic size of the gel fibrils seems to depend only slightly on the alginate concentration. Second, limited data indicate that the polymer composition $\left(F_{\mathrm{G}}\right)$ seems 


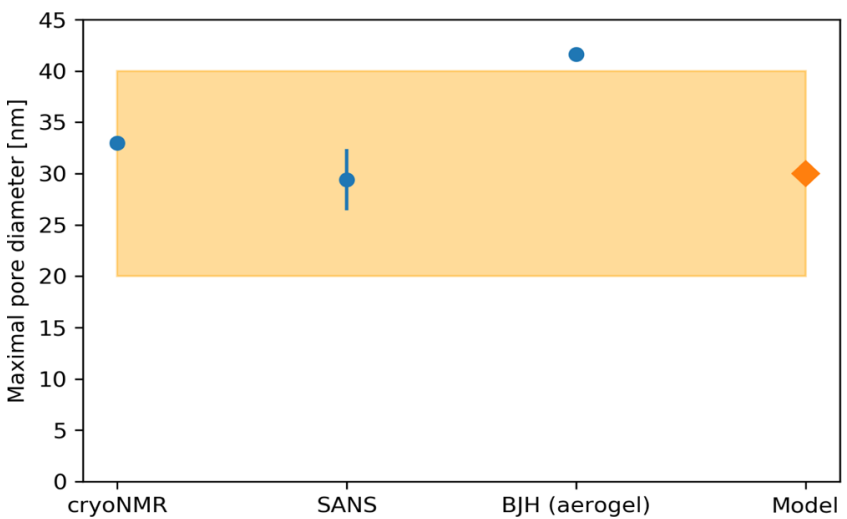

Figure 13. Maximal pore diameter determined by different experimental techniques compared with the model prediction (diamond symbol and the color band).

to have an impact leading to larger fibrils for lower $F_{\mathrm{G}}$. Third, the fibril size strongly depends on the ion concentration $f$ with oversaturated gels in the range of 10-20 nm (also at lower $F_{\mathrm{G}}$ ), while lower ion concentrations (sometimes near estimated saturation) do not exceed $10 \mathrm{~nm}$ (largely at higher $F_{\mathrm{G}}$ ), with an overwhelming majority of the data at $2-3 \mathrm{~nm}$. In fact, Stokke et al. ${ }^{75}$ had also noticed a strong correlation between the characteristic size and the ratio $\left[\mathrm{Ca}^{2+}\right] /[\mathrm{G}]$. It should be stressed that only limited data with a large variability are available for the case $f \simeq 0.5$, see below. Further commenting on the SAXS data, we note that the fibril size of $2-3 \mathrm{~nm}$ was measured when calcium was added in a controlled way (via dispersing or dissolving a known amount of Ca-containing precursors). In the material science, it is rather unusual to control the $\mathrm{Ca}$ content. In fact, quite concentrated $\mathrm{CaCl}_{2}$ solutions are often employed (e.g., $0.72 \mathrm{M}$ which corresponds to $c a .11 \mathrm{wt} \%$, see ref 73) with no control of the final calcium content in the resulting hydrogel. Much larger fibril sizes (10$20 \mathrm{~nm}$ ) are reported for these oversaturated cases. We arbitrarily assigned an $f$ of 1.0 to such cases in Table 5 .

The simulation results at $c=1 \mathrm{wt} \%, M_{\mathrm{w}}=155 \mathrm{kDa}$, and $f=0.5$ showed fibril/bundle diameters between $0.6 \mathrm{~nm}$ (single fibers) and $5.8 \mathrm{~nm}$, see Section 3.6 for analysis methodology. Average fibril diameters over all dimer units were $1.1 \pm 0.5 \mathrm{~nm}$ including single fibers and $1.5 \pm 0.3 \mathrm{~nm}$ excluding single fibers. Consequently, a large number of fibers remain nonaggregated in the solution, which is attributed to a high degree of homogeneity and restraint of single fibers due to cross-linking. However, this analysis is highly dependent on the definition of the maximum distance between two fibers to be considered a fibril ( $0.9 \mathrm{~nm}$ between dimer COM in Section 3.6). This distance remains difficult to define in the context of SAXS and when increasing it, for example, to $1.5 \mathrm{~nm}$, the average fibril diameter increases to $1.7 \pm 1.1 \mathrm{~nm}$ including single fibers and 2.4 $\pm 0.8 \mathrm{~nm}$ when excluding single fibers. Overall, the simulation results are at the lower end in comparison with SAXS data. The most similar experimental conditions by Stokke et al. ${ }^{75}\left(\operatorname{InG}_{155}\right.$ with only a slightly lower $F_{\mathrm{G}}=0.53$ and $f=0.44$ ) found fibril diameters between 1.96 and $4.76 \mathrm{~nm}\left(R_{1}-R_{2}\right)$, which is slightly higher but in reasonable agreement with simulation results in the context of multiplicity of junction zones in SAXS. ${ }^{75}$

The scattering profile is often analyzed using the Guinier approximation, leading most prominently to multiplicity of junction zones, ${ }^{75}$ that is, high density/thickness zones, as well as having been found challenging to apply due to curvature effects
Table 5. Summary of SAXS Measurements for Ca-CrossLinked Hydrogels and Aerogels

\begin{tabular}{|c|c|c|c|c|c|}
\hline $\begin{array}{c}\text { alginate conc. } \\
{[\mathrm{wt} \%]}\end{array}$ & $f[-]$ & $F_{\mathrm{G}}[-]$ & $\begin{array}{c}M_{\mathrm{w}} \\
{[\mathrm{kDa}]}\end{array}$ & $\begin{array}{c}\text { fibril diameter } \\
{[\mathrm{nm}]}\end{array}$ & refs \\
\hline 2 & $1.0^{a}$ & 0.42 & 250 & 11.6 & 73 \\
\hline 3 & $1.0^{a}$ & 0.42 & 250 & 12.8 & 73 \\
\hline 5 & $1.0^{a}$ & 0.42 & 250 & 14.2 & 73 \\
\hline 2 & $1.0^{a}$ & 0.35 & n.d. & 10.2 & 70 \\
\hline 1 & $1.0^{a}$ & 0.35 & n.d. & 9.8 & 71 \\
\hline 2 & 0.46 & 0.63 & n.d. & $6.7 \pm 0.3$ & 66 \\
\hline 2 & 0.46 & 0.63 & n.d. & $9.1 \pm 0.3^{d}$ & 66 \\
\hline 2 & 0.52 & 0.33 & n.d. & $21.8 \pm 0.3$ & 66 \\
\hline 2 & 0.52 & 0.33 & n.d. & $15.4 \pm 0.3^{d}$ & 66 \\
\hline $1.0^{b}$ & 0.175 & 0.68 & 160 & $2.2^{c}$ & 74 \\
\hline $1.0^{b}$ & 0.175 & 0.39 & 230 & $1.4^{c}$ & 74 \\
\hline $0.5^{b}$ & 0.263 & 0.53 & 155 & $2.9^{e}$ & 75 \\
\hline $0.5^{b}$ & 0.525 & 0.53 & 155 & $3.6^{e}$ & 75 \\
\hline $0.75^{b}$ & 0.263 & 0.53 & 155 & $2.6^{e}$ & 75 \\
\hline $0.55^{b}$ & 0.350 & 0.53 & 155 & $3.4^{e}$ & 75 \\
\hline $1.0^{b}$ & 0.175 & 0.39 & 230 & $2.0^{e}$ & 75 \\
\hline $1.0^{b}$ & 0.175 & 0.39 & 230 & $2.2^{e}$ & 75 \\
\hline $1.0^{b}$ & 0.350 & 0.39 & 230 & $2.5^{e}$ & 75 \\
\hline $1.0^{b}$ & 0.175 & 0.53 & 155 & $2.2^{e}$ & 75 \\
\hline $1.0^{b}$ & 0.350 & 0.53 & 155 & $2.9^{e}$ & 75 \\
\hline $1.0^{b}$ & 0.131 & 0.53 & 155 & $1.5^{e}$ & 75 \\
\hline $1.0^{b}$ & 0.175 & 0.53 & 155 & $2.3^{e}$ & 75 \\
\hline $1.0^{b}$ & 0.219 & 0.53 & 155 & $2.5^{e}$ & 75 \\
\hline $1.0^{b}$ & 0.263 & 0.53 & 155 & $2.8^{e}$ & 75 \\
\hline $1.0^{b}$ & 0.438 & 0.53 & 155 & $3.4^{e}$ & 75 \\
\hline $1.25^{b}$ & 0.210 & 0.53 & 155 & $2.4^{e}$ & 75 \\
\hline $1.25^{b}$ & 0.259 & 0.53 & 155 & $2.9^{e}$ & 75 \\
\hline $1.5^{b}$ & 0.175 & 0.53 & 155 & $2.3^{e}$ & 75 \\
\hline $0.5^{b}$ & 0.175 & 0.50 & 455 & $2.2^{e}$ & 75 \\
\hline $0.5^{b}$ & 0.350 & 0.50 & 455 & $2.7^{e}$ & 75 \\
\hline $1.0^{b}$ & 0.175 & 0.50 & 455 & $2.1^{e}$ & 75 \\
\hline $1.0^{b}$ & 0.350 & 0.50 & 455 & $2.9^{e}$ & 75 \\
\hline $0.5^{b}$ & 0.175 & 0.50 & 455 & $1.6^{e}$ & 75 \\
\hline $1.0^{b}$ & 0.175 & 0.70 & 51 & $3.5^{e}$ & 75 \\
\hline $1.0^{b}$ & 0.350 & 0.70 & 51 & $4.4^{e}$ & 75 \\
\hline $1^{b}$ & 0.175 & 0.68 & 160 & $2.5^{e}$ & 75 \\
\hline $1^{b}$ & 0.525 & 0.68 & 160 & $3.2^{e}$ & 75 \\
\hline $1^{b}$ & 0.175 & 0.68 & 160 & $2.9^{e}$ & 75 \\
\hline $1^{b}$ & 0.350 & 0.68 & 160 & $3.6^{e}$ & 75 \\
\hline $1^{b}$ & 0.525 & 0.68 & 160 & $6.0^{e}$ & 75 \\
\hline $0.5^{b}$ & 0.175 & 0.66 & 465 & $2.1^{e}$ & 75 \\
\hline $0.5^{b}$ & 0.350 & 0.66 & 465 & $2.9^{e}$ & 75 \\
\hline $1^{b}$ & 0.175 & 0.66 & 465 & $2.4^{e}$ & 75 \\
\hline $1^{b}$ & 0.35 & 0.66 & 465 & $3.1^{e}$ & 75 \\
\hline $1^{b}$ & 0.175 & 0.66 & 465 & $1.9^{e}$ & 75 \\
\hline
\end{tabular}

${ }^{a} f$ of 1.0 is assumed, given a large excess of $\mathrm{Ca}^{2+}$ over alginate, but not directly measured in the cited paper. ${ }^{b}$ Calculated from alginate concentration in $\mathrm{g} / \mathrm{L}$, assuming density of the alginate solution of $1 \mathrm{~g} /$ $\mathrm{cm}^{3} .{ }^{c}$ Estimated from: $R_{1}=8.0 \AA$ with the corresponding weights from the cited work. ${ }^{d}$ Data for aerogels derived from the corresponding alginate solution. ${ }^{e}$ Averaged from two values of the characteristic size given in the original publication.

of Guinier plots for high-G and high-calcium contents. ${ }^{75}$ In fact, high-G and high-calcium contents are favorable for gelation. Consequently, a direct comparison is difficult in this context. In order to make the data more comparable, in-depth modeling of the scattering profile for the simulated structures would be necessary, which is beyond the scope of this work. The 
presented simulation approach consists of an ideally homogeneous polymer solution, leading to an ideal polymer network with the remaining individual fibers, which are constrained within the network. Real-world samples likely contain a variety of inhomogeneities such as zones with gel density gradients (e.g., due to concentration of the polymer at the gas/liquid interface of entrapped gas bubbles and the presence of low-molecularweight alginate chains). These factors may lead to a local breakup of the network and in turn to thicker fibrils.

\section{CONCLUSIONS AND OUTLOOK}

In conclusion, a mesoscale modeling approach based on Langevin dynamics for the gelation of polymers has been presented. As a model system, the alginate polymer system using calcium cations in aqueous media was selected. The entire model was parameterized using literature data and theoretical considerations. The alginate polymer was abstracted using dimers as the smallest unit (i.e., particle) leading to three different units and polymer fibers modeled using a bonded particle approach. Diffusion was modeled using a previously developed diffusion model. ${ }^{39}$ The LJ potential was chosen as a basic potential shape for unbonded interaction and a probabilistic model implemented to model the binding and unbinding of calcium ions of which the unbonded interaction is dependent.

The impact of various composition and process parameters on gelation was investigated including the ion concentration, polymer concentration, composition of alginate, and molecular weight of polymer fibers. Additionally, the impact of simulation procedures such as annealing and unbinding probabilities was investigated. The model was verified against own and the literature experimental data for bundle size and PSD. The model reproduces both the quantities in reasonable agreement with experiments.

Overall, the model provides a framework and step toward mesoscale modeling of the gelation of polymers. Using the model, gelation can be numerically investigated on the $\mu \mathrm{m}$ scale, which is especially important to identify mechanisms during gelation and can be used complementary to regular MD, which provides lower-scale insights. Moving forward, the model can be extended to capture heterogeneous calcium distributions, for example, using a finite-volume approach. Additionally, the binding and unbinding probability model of ions could be further improved by MD simulations. Lastly, alternative methods to simulated annealing, such as the replica exchange method, could be further employed.

Overall, it was shown that the proposed strategy and developed models allow us to appropriately describe the desired structural formation in the context of gelation on relative large time and length scales.

\section{DATA AND SOFTWARE AVAILABILITY}

The DEM code MUSEN ${ }^{43}$ used for this work is open-source and freely available at https://github.com/msolids/musen. Any modifications have been described in detail including their implementation in Section 3, and all parameters are listed in Tables 1-3. Time-dependent analysis data of all simulation case studies are provided in the Supporting Information. Binary data are available upon request due to their significant size (>1 TB).

\section{ASSOCIATED CONTENT}

\section{(s) Supporting Information}

The Supporting Information is available free of charge at https://pubs.acs.org/doi/10.1021/acs.jcim.1c01076.

Markov chain parameters, time-dependent data of all simulation case studies, chord length analysis of gel pores at the end of each simulation case, solid-state NMR data, and pore size literature (PDF)

\section{AUTHOR INFORMATION}

\section{Corresponding Author}

Philipp Nicolas Depta - Institute of Solids Process Engineering and Particle Technology, Hamburg University of Technology, 21073 Hamburg, Germany; 이이.org/0000-0003-05795220; Email: nicolas.depta@tuhh.de

\section{Authors}

Pavel Gurikov - Laboratory for Development and Modeling of Novel Nanoporous Materials, Hamburg University of Technology, 21073 Hamburg, Germany

Baldur Schroeter - Institute for Thermal Separation Processes, Hamburg University of Technology, 21073 Hamburg, Germany; (1) orcid.org/0000-0002-2577-055X

Attila Forgács - Department of Inorganic and Analytical Chemistry, MTA-DE Redox and Homogeneous Catalytic Reaction Mechanisms Research Group, University of Debrecen, H-4032 Debrecen, Hungary

József Kalmár - Department of Inorganic and Analytical Chemistry, MTA-DE Redox and Homogeneous Catalytic Reaction Mechanisms Research Group, University of Debrecen, H-4032 Debrecen, Hungary; () orcid.org/0000-0002-24226106

Geo Paul - Department of Science and Technological Innovation, Universitá del Piemonte Orientale, 15121 Alessandria, Italy; 이이.orcid/0000-0002-0944-0016

Leonardo Marchese - Department of Science and Technological Innovation, Universitá del Piemonte Orientale, 15121 Alessandria, Italy; 이이. orcid.org/0000-0001-91911237

Stefan Heinrich - Institute of Solids Process Engineering and Particle Technology, Hamburg University of Technology, 21073 Hamburg, Germany

Maksym Dosta - Institute of Solids Process Engineering and Particle Technology, Hamburg University of Technology, 21073 Hamburg, Germany

Complete contact information is available at: https://pubs.acs.org/10.1021/acs.jcim.1c01076

\section{Notes}

The authors declare no competing financial interest.

\section{ACKNOWLEDGMENTS}

The authors thank Prof. Simcha Srebnik for providing the atomistic reference structures as used in ref 32 . Sodium alginate was kindly donated by Nathalie Tanchoux (Institut Charles Gerhardt Montpellier, France). The authors from SPE gratefully acknowledge the German Research Foundation (DFG) for financial support of personal (HE 4526/19-2 within the priority program SPP 1934). P.G. gratefully acknowledges support for this research from the German Research Foundation (DFG) under the project GU 1842/3-1. Assistance of Parnpailin Jeansathawong in the preparation of hydrogels is gratefully 
acknowledged. We are deeply grateful for the SANS measurements to Adél Len (Centre for Energy Research, Hungary) and Zoltán Dudás (Centre for Energy Research, Hungary). The NMR measurements have been financially supported by the National Research, Development and Innovation Office, Hungarian Science Foundation (OTKA: FK_17-124 571). J.K. is grateful for the János Bolyai Research Scholarship of the Hungarian Academy of Sciences.

\section{REFERENCES}

(1) Zhao, S.; Malfait, W. J.; Guerrero-Alburquerque, N.; Koebel, M. M.; Nyström, G. Biopolymer Aerogels and Foams: Chemistry, Properties, and Applications. Angew. Chem., Int. Ed. 2018, 57, 75807608 .

(2) Smirnova, I.; Gurikov, P. Aerogels in Chemical Engineering: Strategies Toward Tailor-Made Aerogels. Annu. Rev. Chem. Biomol. Eng. 2017, 8, 307-334.

(3) Budtova, T.; Aguilera, D. A.; Beluns, S.; Berglund, L.; Chartier, C.; Espinosa, E.; Gaidukovs, S.; Klimek-Kopyra, A.; Kmita, A.; Lachowicz, D.; Liebner, F.; Platnieks, O.; Rodríguez, A.; Tinoco Navarro, L. K.; Zou, F.; Buwalda, S. J. Biorefinery Approach for Aerogels. Polymers 2020, 12, 2779.

(4) Gurikov, P.; Raman, S.; Griffin, J. S.; Steiner, S. A.; Smirnova, I. 110th Anniversary: Solvent Exchange in the Processing of Biopolymer Aerogels: Current Status and Open Questions. Ind. Eng. Chem. Res. 2019, 58, 18590-18600.

(5) Şahin, I.; Uzunlar, E.; Erkey, C. Investigation of the Effect of Gel Properties on Supercritical Drying Kinetics of Ionotropic Alginate Gel Particles. J. Supercrit. Fluids 2019, 152, 104571.

(6) Selmer, I.; Behnecke, A.-S.; Quiño, J.; Braeuer, A. S.; Gurikov, P.; Smirnova, I. Model Development for Sc-Drying Kinetics of Aerogels: Part 1. Monoliths and Single Particles. J. Supercrit. Fluids 2018, 140, 415-430.

(7) Selmer, I.; Behnecke, A.-S.; Farrell, P.; Bueno, A.; Gurikov, P.; Smirnova, I. Model Development for Sc-Drying Kinetics of Aerogels: Part 2. Packed Bed of Spherical Particles. J. Supercrit. Fluids 2019, 147, 149-161.

(8) Ratke, L.; Gurikov, P. The Chemistry and Physics of Aerogels: Synthesis, Processing, and Properties; Cambridge University Press: Cambridge, New York, NY, 2021.

(9) Nakano, A.; Bi, L.; Kalia, R. K.; Vashishta, P. Structural Correlations in Porous Silica: Molecular Dynamics Simulation on a Parallel Computer. Phys. Rev. Lett. 1993, 71, 85-88.

(10) Pereyra, R. G.; Al-Maadeed, M. A.; Carignano, M. A. Modeling Polymeric Gels: The Role of Chain Fexibility on the Structure of Physical Gels. eXPRESS Polym. Lett. 2017, 11, 199-208.

(11) Padmanabhan, V.; Kumar, S. K. Gelation in Semiflexible Polymers. J. Chem. Phys. 2011, 134, 174902.

(12) Nguyen, H. T.; Graham, A. L.; Koenig, P. H.; Gelb, L. D. Computer Simulations of Colloidal Gels: How Hindered Particle Rotation Affects Structure and Rheology. Soft Matter 2020, 16, 256269.

(13) Gonçalves, W.; Morthomas, J.; Chantrenne, P.; Perez, M.; Foray, G.; Martin, C. L. Elasticity and Strength of Silica Aerogels: A Molecular Dynamics Study on Large Volumes. Acta Mater. 2018, 145, 165-174.

(14) Patil, S. P.; Rege, A.; Itskov, M.; Markert, B. Fracture of Silica Aerogels: An All-Atom Simulation Study. J. Non-Cryst. Solids 2018, 498, 125-129.

(15) Patil, S. P.; Parale, V. G.; Park, H.-H.; Markert, B. Mechanical Modeling and Simulation of Aerogels: A Review. Ceram. Int. 2021, 47, 2981-2998.

(16) Abdusalamov, R.; Scherdel, C.; Itskov, M.; Milow, B.; Reichenauer, G.; Rege, A. Modeling and Simulation of the Aggregation and the Structural and Mechanical Properties of Silica Aerogels. J. Phys. Chem. B 2021, 125, 1944-1950.

(17) Hoogerbrugge, P. J.; Koelman, J. M. V. A. Simulating Microscopic Hydrodynamic Phenomena with Dissipative Particle Dynamics. Europhys. Lett. 1992, 19, 155-160.
(18) Pieczywek, P. M.; Płaziński, W.; Zdunek, A. Dissipative Particle Dynamics Model of Homogalacturonan Based on Molecular Dynamics Simulations. Sci. Rep. 2020, 10, 14691.

(19) Pieczywek, P. M.; Cieśla, J.; Płaziński, W.; Zdunek, A. Aggregation and Weak Gel Formation by Pectic Polysaccharide Homogalacturonan. Carbohydr. Polym. 2021, 256, 117566.

(20) Berendsen, H. J. Simulating the Physical World: Hierarchical Modeling from Quantum Mechanics to Fluid Dynamics; Cambridge University Press, 2007.

(21) Guesnet, E.; Bénane, B.; Jauffrès, D.; Martin, C. L.; Baeza, G. P.; Foray, G.; Meille, S.; Olagnon, C.; Yrieix, B. Why Fumed and Precipitated Silica Have Different Mechanical Behavior: Contribution of Discrete Element Simulations. J. Non-Cryst. Solids 2019, 524, 119646.

(22) Dosta, M.; Jarolin, K.; Gurikov, P. Modelling of Mechanical Behavior of Biopolymer Alginate Aerogels Using the Bonded-Particle Model. Molecules 2019, 24, 2543.

(23) Rege, A.; Schestakow, M.; Karadagli, I.; Ratke, L.; Itskov, M. Micro-Mechanical Modelling of Cellulose Aerogels from Molten Salt Hydrates. Soft Matter 2016, 12, 7079-7088.

(24) Chandrasekaran, R.; Hillgärtner, M.; Ganesan, K.; Milow, B.; Itskov, M.; Rege, A. Computational Design of Biopolymer Aerogels and Predictive Modelling of Their Nanostructure and Mechanical Behaviour. Sci. Rep. 2021, 11, 10198.

(25) Grant, G. T.; Morris, E. R.; Rees, D. A.; Smith, P. J. C.; Thom, D. Biological Interactions Between Polysaccharides and Divalent Cations: The Egg-Box Model. FEBS Lett. 1973, 32, 195-198.

(26) Cao, L.; Lu, W.; Mata, A.; Nishinari, K.; Fang, Y. Egg-Box ModelBased Gelation of Alginate and Pectin: A Review. Carbohydr. Polym. 2020, 242, 116389.

(27) Braccini, I.; Pérez, S. Molecular Basis of Ca2+-Induced Gelation in Alginates and Pectins: The Egg-Box Model Revisited. Biomacromolecules 2001, 2, 1089-1096.

(28) Plazinski, W. Molecular Basis of Calcium Binding by Polyguluronate Chains. Revising the Egg-Box Model. J. Comput. Chem. 2011, 32, 2988-2995.

(29) Plazinski, W.; Drach, M. Calcium- $\alpha$-L-Guluronate Complexes: Ca2+ Binding Modes from DFT-MD Simulations. J. Phys. Chem. B 2013, 117, 12105-12112.

(30) Wolnik, A.; Albertin, L.; Charlier, L.; Mazeau, K. Probing the Helical Forms of Ca2+-Guluronan Junction Zones in Alginate Gels by Molecular Dynamics 1: Duplexes. Biopolymers 2013, 99, 562-571.

(31) Stewart, M. B.; Gray, S. R.; Vasiljevic, T.; Orbell, J. D. Exploring the Molecular Basis for the Metal-Mediated Assembly of Alginate Gels. Carbohydr. Polym. 2014, 102, 246-253.

(32) Hecht, H.; Srebnik, S. Structural Characterization of Sodium Alginate and Calcium Alginate. Biomacromolecules 2016, 17, 21602167.

(33) Hecht, H.; Srebnik, S. Sequence-Dependent Association of Alginate with Sodium and Calcium Counterions. Carbohydr. Polym. 2017, 157, 1144-1152.

(34) Hernández Cifre, J. G.; de la Torre, J. G. Ionic Strength Effect in Polyelectrolyte Dilute Solutions Within the Debye-Hückel Approximation: Monte Carlo and Brownian Dynamics Simulations. Polym. Bull. 2014, 71, 2269-2285.

(35) Donati, I.; Paoletti, S. In Alginates: Biology and Applications; Rehm, B. H. A., Ed.; Springer Berlin Heidelberg: Berlin, Heidelberg, 2009; Vol. 13, pp 1-53.

(36) Fang, Y.; Al-Assaf, S.; Phillips, G. O.; Nishinari, K.; Funami, T.; Williams, P. A.; Li, L. Multiple Steps and Critical Behaviors of the Binding of Calcium to Alginate. J. Phys. Chem. B 2007, 111, 24562462.

(37) Potyondy, D. O. The Bonded-Particle Model as a Tool for Rock Mechanics Research and Application: Current Trends and Future Directions. Geosyst. Eng. 2015, 18, 1-28.

(38) Rybczyński, S.; Dosta, M.; Schaan, G.; Ritter, M.; Schmidt-Döhl, F. Numerical Study on the Mechanical Behavior of Ultrahigh Performance Concrete Using a Three-Phase Discrete Element Model. Struct. Concr. 2020, 1-16. 
(39) Depta, P. N.; Jandt, U.; Dosta, M.; Zeng, A.-P.; Heinrich, S. Toward Multiscale Modeling of Proteins and Bioagglomerates: An Orientation-Sensitive Diffusion Model for the Integration of Molecular Dynamics and the Discrete Element Method. J. Chem. Inf. Model. 2019, 59, 386-398.

(40) Smit, B.; Frenkel, D. Vapor-Liquid Equilibria of the TwoDimensional Lennard-Jones Fluid(s). J. Chem. Phys. 1991, 94, 56635668.

(41) Steinbach, P. J.; Brooks, B. R. New Spherical-Cutoff Methods for Long-Range Forces in Macromolecular Simulation. J. Comput. Chem. 1994, 15, 667-683.

(42) Kirkpatrick, S.; Gelatt, C. D.; Vecchi, M. P. Optimization by Simulated Annealing. Science 1983, 220, 671-680.

(43) Dosta, M.; Skorych, V. MUSEN: An Open-Source Framework for GPU-Accelerated DEM Simulations. SoftwareX 2020, 12, 100618.

(44) NVIDIA Corporation. CUDA Toolkit V11.2 Programming Guide; NVIDIA Corporation, 2021.

(45) Marsaglia, G. Xorshift RNGs. J. Stat. Softw. 2003, 8, 1-6.

(46) Shoemake, K. Graphics Gems III (IBM Version); Elsevier, 1992; pp 124-132.

(47) Preibisch, I.; Niemeyer, P.; Yusufoglu, Y.; Gurikov, P.; Milow, B.; Smirnova, I. Polysaccharide-Based Aerogel Bead Production via Jet Cutting Method. Materials 2018, 11, 1287.

(48) López-Iglesias, C.; Barros, J.; Ardao, I.; Gurikov, P.; Monteiro, F. J.; Smirnova, I.; Alvarez-Lorenzo, C.; García-González, C. A. Jet Cutting Technique for the Production of Chitosan Aerogel Microparticles Loaded with Vancomycin. Polymers 2020, 12, 273.

(49) Paul, G.; Boccaleri, E.; Cassino, C.; Gastaldi, D.; Buzzi, L.; Canonico, F.; Marchese, L. Fingerprinting the Hydration Products of Hydraulic Binders Using Snapshots from Time-Resolved in Situ Multinuclear MAS NMR Spectroscopy. J. Phys. Chem. C 2021, 125, 9261-9272.

(50) Massiot, D.; Fayon, F.; Capron, M.; King, I.; Le Calvé, S.; Alonso, B.; Durand, J.-O.; Bujoli, B.; Gan, Z.; Hoatson, G. Modelling One- and Two-Dimensional Solid-State NMR Spectra: Modelling 1D and 2D Solid-State NMR Spectra. Magn. Reson. Chem. 2002, 40, 70-76.

(51) Forgács, A.; Papp, V.; Paul, G.; Marchese, L.; Len, A.; Dudás, Z.; Fábián, I.; Gurikov, P.; Kalmár, J. Mechanism of Hydration and Hydration Induced Structural Changes of Calcium Alginate Aerogel. ACS Appl. Mater. Interfaces 2021, 13, 2997-3010.

(52) Salomonsen, T.; Jensen, H. M.; Larsen, F. H.; Steuernagel, S.; Engelsen, S. B. Alginate Monomer Composition Studied by Solutionand Solid-State NMR-a Comparative Chemometric Study. Food Hydrocolloids 2009, 23, 1579-1586.

(53) Allen, S. G.; Stephenson, P. C. L.; Strange, J. H. Internal Surfaces of Porous Media Studied by Nuclear Magnetic Resonance Cryoporometry. J. Chem. Phys. 1998, 108, 8195-8198.

(54) Dore, J. C.; Webber, J. B. W.; Strange, J. H. Characterisation of Porous Solids Using Small-Angle Scattering and NMR Cryoporometry. Colloids Surf., A 2004, 241, 191-200.

(55) Petrov, O. V.; Furó, I. NMR Cryoporometry: Principles, Applications and Potential. Prog. Nucl. Magn. Reson. Spectrosc. 2009, $54,97-122$.

(56) Kalmár, J.; Kéri, M.; Erdei, Z.; Bányai, I.; Lázár, I.; Lente, G.; Fábián, I. The Pore Network and the Adsorption Characteristics of Mesoporous Silica Aerogel: Adsorption Kinetics on a Timescale of Seconds. RSC Adv. 2015, 5, 107237-107246.

(57) Ammann, C.; Meier, P.; Merbach, A. A Simple Multinuclear NMR Thermometer. J. Magn. Reson. 1982, 46, 319-321.

(58) Kéri, M.; Forgács, A.; Papp, V.; Bányai, I.; Veres, P.; Len, A.; Dudás, Z.; Fábián, I.; Kalmár, J. Gelatin Content Governs Hydration Induced Structural Changes in Silica-Gelatin Hybrid Aerogels Implications in Drug Delivery. Acta Biomater. 2020, 105, 131-145.

(59) Lázár, I.; Forgács, A.; Horváth, A.; Király, G.; Nagy, G.; Len, A.; Dudás, Z.; Papp, V.; Balogh, Z.; Moldován, K.; Juhász, L.; Cserháti, C.; Szántó, Z.; Fábián, I.; Kalmár, J. Mechanism of Hydration of Biocompatible Silica-Casein Aerogels Probed by NMR and SANS Reveal Backbone Rigidity. Appl. Surf. Sci. 2020, 531, 147232.
(60) Beaucage, G. Approximations Leading to a Unified Exponential/ Power-Law Approach to Small-Angle Scattering. J. Appl. Crystallogr. 1995, 28, 717-728.

(61) Hammouda, B. Analysis of the Beaucage Model. J. Appl. Crystallogr. 2010, 43, 1474-1478.

(62) Weast, R. CRC Handbook of Chemistry and Physics, 64th ed.; CRC Press, 1983.

(63) Cerdà, J. J.; Sintes, T.; Chakrabarti, A. Excluded Volume Effects on Polymer Chains Confined to Spherical Surfaces. Macromolecules 2005, 38, 1469-1477.

(64) Shannon, R. D. Revised Effective Ionic Radii and Systematic Studies of Interatomic Distances in Halides and Chalcogenides. Acta Crystallogr., Sect. A: Cryst. Phys., Diffr., Theor. Gen. Crystallogr. 1976, 32, 751-767.

(65) Ribeiro, A. C. F.; Barros, M. C. F.; Teles, A. S. N.; Valente, A. J. M.; Lobo, V. M. M.; Sobral, A. J. F. N.; Esteso, M. A. Diffusion Coefficients and Electrical Conductivities for Calcium Chloride Aqueous Solutions at 298.15K and 310.15K. Electrochim. Acta 2008, 54, 192-196.

(66) Agulhon, P.; Robitzer, M.; David, L.; Quignard, F. Structural Regime Identification in Ionotropic Alginate Gels: Influence of the Cation Nature and Alginate Structure. Biomacromolecules 2012, 13, 215-220.

(67) Lindahl, E.; Abraham, M. J.; Hess, B.; Van Der Spoel, D. GROMACS 2020.1 Manual; Zendo, 2020,

(68) Rodriguez-Cruz, S. E.; Jockusch, R. A.; Williams, E. R. Hydration Energies of Divalent Metal Ions, $\mathrm{Ca}^{2+}\left(\mathrm{H}_{2} \mathrm{O}\right)_{n}(n=5-7)$ and $\mathrm{Ni}^{2+}$ $\left(\mathrm{H}_{2} \mathrm{O}\right)_{n} \quad(n=6-8)$, Obtained by Blackbody Infrared Radiative Dissociation. J. Am. Chem. Soc. 1998, 120, 5842-5843.

(69) Lu, L.; Liu, X.; Dai, L.; Tong, Z. Difference in Concentration Dependence of Relaxation Critical Exponent $\mathrm{n}$ for Alginate Solutions at Sol-Gel Transition Induced by Calcium Cations. Biomacromolecules 2005, 6, 2150-2156.

(70) Robitzer, M.; David, L.; Rochas, C.; Di Renzo, F.; Quignard, F. Nanostructure of Calcium Alginate Aerogels Obtained from Multistep Solvent Exchange Route. Langmuir 2008, 24, 12547-12552.

(71) Robitzer, M.; David, L.; Rochas, C.; Di Renzo, F.; Quignard, F. Supercritically-Dried Alginate Aerogels Retain the Fibrillar Structure of the Hydrogels. Macromol. Symp. 2008, 273, 80-84.

(72) Kline, S. R. Reduction and Analysis of SANS and USANS Data Using IGOR Pro. J. Appl. Crystallogr. 2006, 39, 895-900.

(73) Maki, Y.; Ito, K.; Hosoya, N.; Yoneyama, C.; Furusawa, K.; Yamamoto, T.; Dobashi, T.; Sugimoto, Y.; Wakabayashi, K. Anisotropic Structure of Calcium-Induced Alginate Gels by Optical and SmallAngle X-Ray Scattering Measurements. Biomacromolecules 2011, 12, $2145-2152$.

(74) Yuguchi, Y.; Urakawa, H.; Kajiwara, K.; Draget, K. I.; Stokke, B. T. Small-Angle X-Ray Scattering and Rheological Characterization of Alginate Gels. 2. Time-Resolved Studies on Ionotropic Gels. J. Mol. Struct. 2000, 554, 21-34.

(75) Stokke, B. T.; Draget, K. I.; Smidsrød, O.; Yuguchi, Y.; Urakawa, H.; Kajiwara, K. Small-Angle X-Ray Scattering and Rheological Characterization of Alginate Gels. 1. Ca-Alginate Gels. Macromolecules 2000, 33, 1853-1863. 